\title{
COVID-19 Lesion Segmentation using Lung CT Scan Images: Comparative Study based on Active Contour Models
}

Younes Akbari ( $\nabla$ akbari_younes@yahoo.com )

Qatar University

Hanadi Hassen

Qatar University

Somaya Al-maadeed

Qatar University

Susu M. Zughaier

Qatar University

\section{Research Article}

Keywords: Lung CT scans, COVID-19 infection, Pneumonia, Active contour models, Parametric methods, Level set methods, Region-based models, Edge-based models

Posted Date: July 9th, 2020

DOl: https://doi.org/10.21203/rs.3.rs-40406/v1

License: (c) (i) This work is licensed under a Creative Commons Attribution 4.0 International License. Read Full License

Version of Record: A version of this preprint was published at Applied Sciences on August 30th, 2021. See the published version at https://doi.org/10.3390/app11178039. 


\title{
COVID-19 Lesion Segmentation using Lung CT Scan Images: Comparative Study based on Active Contour Models
}

\author{
Younes Akbari ${ }^{1, *+}$, Hanadi Hassen ${ }^{1,+}$, Somaya Al-maadeed ${ }^{1,+}$, and Susu M. Zughaier ${ }^{2,+}$ \\ ${ }^{1}$ Department of Computer Science and Engineering, Qatar University, Doha, Qatar \\ ${ }^{2}$ College of Medicine, QU Health, Qatar University \\ *corresponding.akbari_younes@semnan.ac.ir \\ +these authors contributed equally to this work
}

\begin{abstract}
Pneumonia is a lung infection threaten that threats all age groups. In this paper, using CT scans images, we used active contour models to evaluate and determine pneumonia infection caused by the Coronavirus disease (COVID-19). A background of active contour models (ACM) including contour representation and object boundary description methods is presented. The focus of this paper is on the conducted works based on the external forces. These methods include edge-based and region-based methods. Furthermore, the explanations of these methods, as well as the advantages and disadvantages of each method are presented. Finally, a comparison between the performances of the conducted works has been done based on a database of Lung CT Scan Images. The present review helps readers identify research starting points in active contour models on COVID19 research, which is a high priority topic to guide researchers and practitioners. In addition, when there are not enough images to use machine learning techniques, such as deep learning methods, the experimental results indicate that active contour methods obtain promising results.
\end{abstract}

\section{Introduction}

COVID-19, is a pandemic viral disease caused by the Severe Acute Respiratory Syndrome Coronavirus 2 (SARS-CoV-2). Current golden standard diagnostic method for COVID-19 cases is via viral nucleic acid detection using Reverse Transcription Polymerase Chain Reaction (RT-PCR). As well as the reduced sensitivity of some tests yielding false negative results, other methods to accurately diagnose COVID19 are highly desired. In addition, this RT-PCR molecular method needs cumbersome operations in highly controlled environment, usually taking about 4 hours to receive the test results, limiting its spread popularization. Notably, the false negative cases of RT-PCR tests are the potential threat to public wellness. To facilitate COVID19 diagnosis, medical radiology imaging is employed as an ultra-fast alternative for discovering the rapidly progressing suspected or asymptomatic cases into viral pneumonia manifesting as severe lung disease. Radiological imaging such as $\mathrm{X}$-rays are usually obtained for patients with clinical symptoms suggesting lung infection. ${ }^{1}$ demonstrated that CT scan test exhibit higher sensitivity than the RT-PCR ones. This point is further validated by, in which CT scans and RT-PCR tests obtain the sensitivity of $98 \%$ and $71 \%$, respectively. However, the diagnosis duration is still the major limitation of CT scan, even experienced radiologists need about 21.5 minutes to analyze the test results of each case ${ }^{1}$.

Therefore, obtaining the region of infection will reduce the time for radiologists to analyze the testing results. Automatic segmentation of the region of infection can be achieved through effective segmentation algorithms. Currently, there are several segmentation algorithms in the literature that showed accurate and robust segmentation results. Generally, they can be classified into four categories, the clustering based ${ }^{2,3}$, the graph-cut-based ${ }^{4,5}$, neural-network-based ${ }^{6,7}$ and the active-contour-based methods $^{8-10}$. The clustering-based methods use clustering algorithms such as Kmeans and fuzzy C-means based on the assumption that each pixel can be assigned to the pixels belonging to the same class since they have specific distribution. The clustering-based methods show high efficiency, however, they are sensitive to initial clustering centers and they need a manual setting of clustering number. In the graph-cut-based methods, pixels correlation is taken into account and the segmentation problem is transformed into a graph partition problem where a cut energy model is constructed then the segmentation curve is the cut that minimizes the energy. The disadvantage of this method is the difficulty of constructing accurate weights for the correlations between pixels, this usually leads to over-segmentation or under-segmentation problems especially in complicated regions. The emergence of neural networks-based solutions for semantic segmentation ${ }^{6,7}$, requires a large set of images to train the network in addition it lacks giving fine segmentation for tiny areas which is very crucial in medical image analysis.

One of the methods that perform stably is the active contour (snake) models (ACM). This method grant closed boundaries 
and have been effective and prevalent. The general idea behind ACMs is applying the partial differential equations (PDEs) to iteratively evolution of the initial contour toward object boundaries by minimizing a given energy functional ${ }^{11,12}$ which is a function of the contour's internal and external energies. According to contour representation (parametric or level set) and object boundary description (edge-based or region-based), ACMs can be classified into four categories including: parametric representation with edge-based description ${ }^{11,13}$, parametric representation with region-based description ${ }^{14,15}$, level set representation with edge-based description ${ }^{16,17}$ and level set representation with region-based description ${ }^{18,19}$. In the parametric ACM, the contour is explicitly represented as polynomials or splines ${ }^{20,21}$. Given an initial contour, the external energies drive the evolution of a parametric ACM while the internal energies maintain the shape of the contour. Parametric ACM has the ability to extract a single object given an input of a single initial contour. Their strength, the parametric snakes have limited capture range which is the region where the external forces are strong enough to drive contour evolution that's why they have to be initialized near the object contour. Another issue is that the parametric snakes are unable to accurately extract concave shapes. In the level set ACMs, contour is implicitly represented in the zero level set. In this method, the deformation of the level set function yields to contour evolution. Compared to the parametric ACMs, level set model can capture multiple objects by single initial contour and complex geometry. However, since in the level set models the deformation of a higher dimensional function is required, these methods are usually slower than parametric methods. Further, for many applications, such as medical image processing it is necessary to extract only a single object ${ }^{22}$ and therefore in such these situations, parametric representation is preferred to the level set representation. To solve the contour evolution equation, every representation scheme needs to select appropriate numerical methods. Finite element method ${ }^{23}$ is used in parametric snake models, whereas the finite difference method ${ }^{24}$ is used in level set models. Object boundary description (boundary-based segmentation) methods based on the external force include region-based and edge-based models. Region-based models use more global information to define object boundaries. To control the evolution, region-based models employ the statistical information of inside and outside the contour ${ }^{18,19}$. In the region-based approach, the deformation of ACM is based on an energy minimization algorithm. In region-based method, many functions have been considered as $\mathrm{ESF}^{19}$ such as signed pressure function (SPF). Edge-based models use image gradient to construct an edge stopping function (ESF) ${ }^{17,20}$ which is used to stop the contour evolution on the object boundaries. Using the ESF, the boundary points can be characterized by a differential property in terms of the image gradient. It should be noted that in order to use the advantages of edge-based and region-based methods and avoid their disadvantages, hybrid description approaches have been recently used ${ }^{25}$. Two important challenges in the applications of ACMs are initialization and convergence. For example, some of parametric ACMs be affected by saddle point and stationary point problems that lead to failure of convergence ${ }^{17}$. ACM models quickly respond to the initial condition, therefore, bad initial contour can give a poor result as demonstrated in Fig. 1 which depicts the initialization of contour outside, overlapping (cross) or inside of the image.

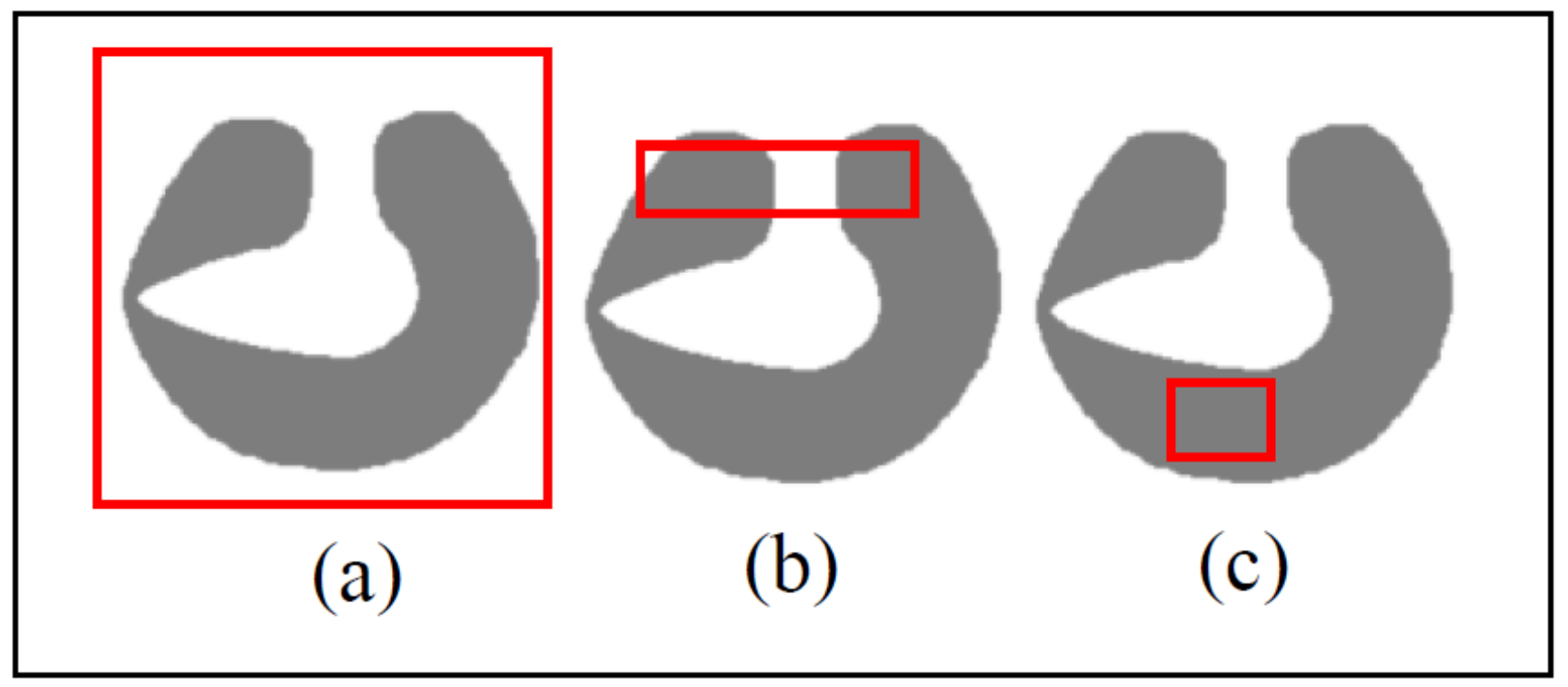

Figure 1. Three examples of initial contours considered outside (a), overlap (b), and inside (c) the image

The region-based methods are more efficient in detecting the exterior and interior boundaries than the edge-based methods because they are less sensitive to the location of initial contour. However, on weak edges and concave shapes edge-based 
methods have more successful performance than region-based descriptions.

This work aims at comparing recent active contour methods used in detecting COVID-19 pneumonia infection using CTSI, as shown in Fig. 2. The infected area is extracted with good accuracy which is computed based on seven measures. Our proposed segmentation experiments are being conducted using COVID-CS database ${ }^{26}$ which contains one hundred of CT COVID-19 images with dimension $512 \times 512 \times 1$ pixels and all the images are associated with the Ground-Truth-Image (GTI). This paper presents the state-of-the-art methods in the categories mentioned above. We selected methods from 2008 to 2020 that obtained the best results on medical images segmentation. Moreover, these methods have been compared in terms of robustness to initialization. This paper focuses on CT COVID-19 image segmentation. The main contributions of this paper are

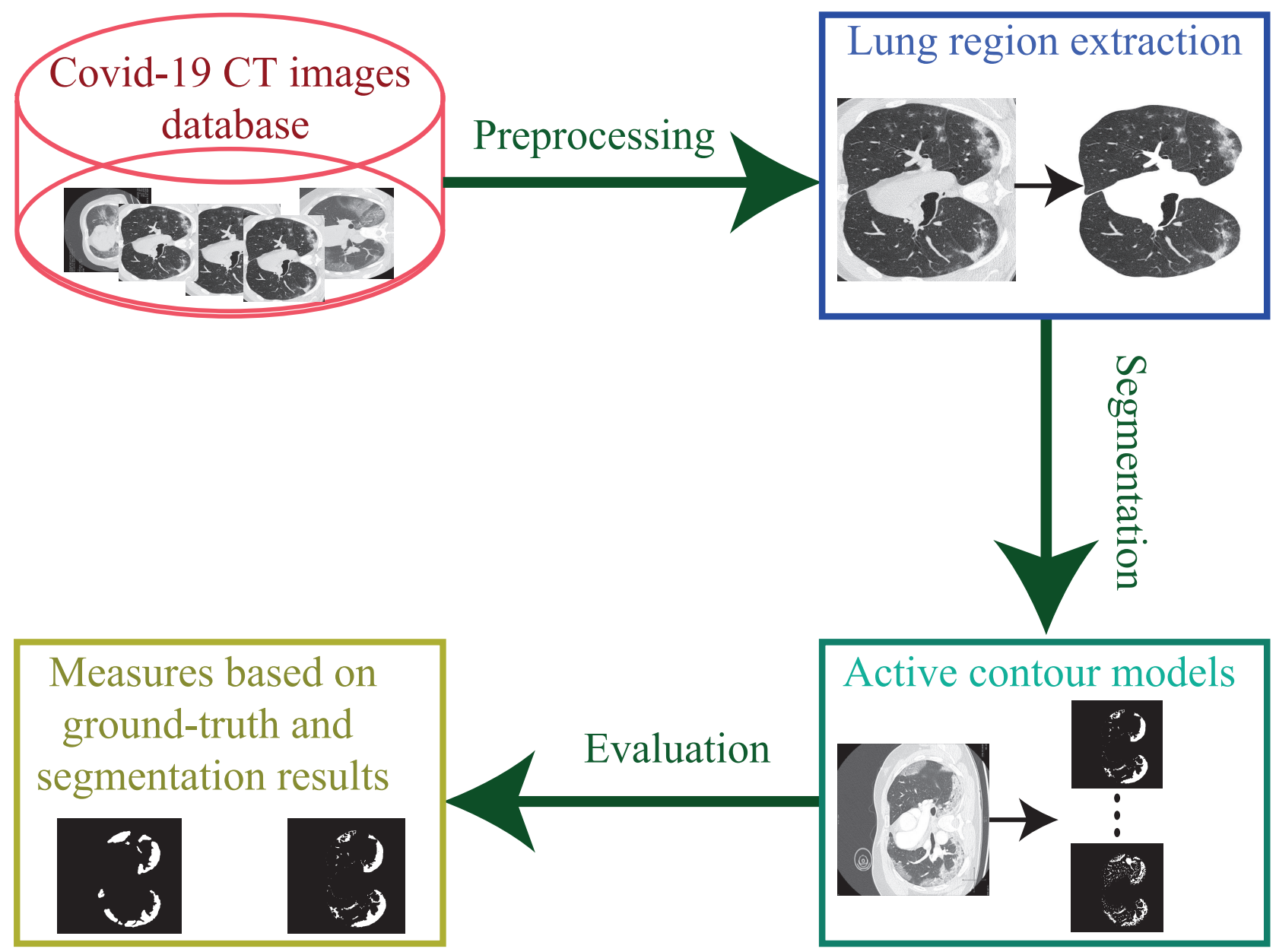

Figure 2. Overview of the proposed steps

described as follow:

- A survey of active contour models.

- Study on COVID-19 as a hot topic: To the best of the authors' knowledge, this work is the first attempt to investigate active contour models and comparison on images of the disease.

- Presenting a way for next researchers: We investigate different methods and demonstrate which one is effective for the topic and what are the problems.

The remainder of this paper is structured as follow. Section 2 presents a literature review of related work, and Sections 3 and 4 introduces the tested models. Experimental results are presented in Section 5 and Section 6 concludes the paper. 


\section{COVID19 Related Applications}

The 2019 novel coronavirus named COVID-19 by World Health Organization is getting lots of attention recently because it is a new kind of coronavirus that is highly contagious and have not been seen among human before. The virus started in China and rapidly spread around countries. China adopted many technologies to suppress the spread of the virus, from applications that localize people who have the virus to unmanned vehicles that deliver medical supplies, sanitizer robots, food making robots and delivery robots. Machine learning scientists are trying to tackle the pandemic by collecting databases and proposing algorithms that learn from them. Significant number of research works are initiated to speed up the diagnosis and predict the spread of the virus, the number of mortalities and the genetic evolution of the virus. This would be potentially helpful for policymakers to understand how the virus spread and to decide the establishment of quarantine areas. In hospitals, a thoracic computed tomography (CT) examination is used as a diagnostic tool in COVID19 lung infections. Developing algorithms for CT image analysis is the current hot research aims to contribute to the automatic detection of the coronavirus. It is also suggested that algorithms that were applied to identify lung cancer and lung collapse using X-ray images will also be useful for identifying abnormal cases from COVID-19 patients.

Compared to the chest X-ray, the CT has more clear information and it offers better judgement accuracy hence, this research work considered only the CT for the examination. Rodriguez-Morales et al. ${ }^{27}$ present a review of the detection and prediction of COVID-19 pneumonia infection. The current procedures used in the identification of the virus require an experienced radiologist, hence automatic detection would be essential to reduce the assessment time for radiologists. The work in ${ }^{28}$ reviews the recent image processing techniques.

The proposed work here aims at extracting COVID-19 infected region from the CTSI using image segmentation. In addition to infected region extraction, we provide the average infection rate using pixel information in the lung section.

We dedicate this section to attempt on segmentation step based on COVID-19 CT images. In the segmentation step, the recent procedures to detect COVID-19 pneumonia using the CTSI are explored in the following. X-ray Images are more easily accessible around the world in comparison with CT images. However, because of the ribs projected onto soft tissues in 2D and thus confounding image contrast, the X-ray images segmentation are even more challenging. Despite the facts and to the best of our knowledge, none of the image segmentation methods for COVID-19 are used on X-ray images. It should be noted that the segmentation methods in COVID-19 applications can be mainly divided into two categories, namely, the lung-region-oriented and the lung-lesion-oriented methods. The lung-region-oriented methods aim to separate lung regions, namely, whole lung and lung lobes, from other (background) regions in CT or X-ray, which is considered as a pre-requisite step in COVID-19 applications ${ }^{29-38}$. The popular segmentation networks for COVID-19 include classic U-Net ${ }^{29-34}$, UNet++ ${ }^{34,35}$, VB-Net ${ }^{36}$. UNet and its variants achieved reasonable segmentation results in the COVID-19 images. For example, Jin et al ${ }^{35}$ used UNet++ network for segmenting lung region from CT images. Then, the damaged area of the lung is separated. This process is challenging because the damaged area may have different shapes and textures. ${ }^{33}$ developed an automatic AI-based analysis of CT images using deep learning approach to classify CT images into coronavirus and non-coronavirus cases. They reused a system applied before for detecting small opacities and nodules within the lung using UNet for segmenting lung images. To detect corona virus abnormalities, they used the $\mathrm{CNN}$ architecture Resnet-50 which consists of 50 layers to classify images into normal and abnormal cases.

Although there is a lot of research employed to automatically detect the COVID-19, the databases used in these researches were not made publicly available to other users or they have not provided a ground truth for their databases. The sudden emergence of the disease may be the reason that well-prepared databases do not exist. As far as we know, the work of ${ }^{1,39,40}$ are the only one that present databases that can be used by others. $\mathrm{In}^{39}$, the segmentation is performed via the watershed transform techniques ${ }^{41}$ with coarse results and limited accuracy. In this work, a diagnosis system by integrating learning based classification and segmentation networks is presented, to provide explainable diagnostic evidence for doctors and improve the user-interactive aspects of the diagnosis process. ${ }^{1}$ present a novel Joint Classification and Segmentation (JCS) system to perform real-time and explainable COVID-19 diagnosis. However the database is still not publicly available. ${ }^{40}$ implements Firefly Algorithm and Shannon Entropy (FA+SE) based multi-threshold to enhance the pneumonia lesion and implements Markov-Random-Field (MRF) segmentation to extract the lesions with better accuracy. The COVID-19 database available in ${ }^{26}$ is considered for the initial assessment.

To evaluate the effectiveness of document image analysis and recognition methods, seven suitable measures are applied in the competitions, that is: Dice, Jaccard, Bfscore, precision, Recall, Iteration and time. If attained the first five measures values are better (closer to unity or nearer to $100 \%$ ), then the implemented disease examination scheme is confirmed as a better procedure. Given $I_{O}$ and $I_{G T}$ represent the output binary image of the segmentation result and binary image of the ground truth, 
respectively, the measures are defined as follow:

$$
\begin{array}{r}
\text { Precision }=\frac{T P}{T P+F P}, \\
\text { Recall }=\frac{T P}{T P+F N}, \\
\text { Jaccard }=\frac{\left|I_{o} \cap I_{g}\right|}{\left|I_{o} \cup I_{g}\right|}, \\
\text { Dice }=\frac{2\left|I_{o} \cap I_{g}\right|}{\left|I_{o}\right|+\left|I_{g}\right|}, \\
\text { Bfscore }=\frac{2 \times \text { Precision } \times \text { Recall }}{\text { Recall }+ \text { Precision }},
\end{array}
$$

where TP, FP and FN are true positive, false positive and false negative rates.

\section{Background of ACMs}

In this section, a background knowledge of ACMs through the briefly explanation about traditional ACM and geometric ACM models is presented.

\subsection{Traditional ACM}

The earliest active contour or snake, which was a parametric representation with edge-based description, was proposed by Kass et al. $(1988)^{20}$. Active contours are declared as an energy minimization process. sThe energy functional is a function of contour's internal energy $\left(E_{\text {int }}\right)$ addition to the external energy $\left(E_{\text {ext }}\right)$ These energies are two functions of the set of points $(x(s), y(s))$, which make up a snake $c(s)=(x(s), y(s))$, The energy functional denoted by $E_{\text {Snake }}$ is computed as follow:

$$
E_{\text {Snake }}=\int_{0}^{1}\left[E_{\text {int }}(c(s))+E_{\text {ext }}(c(s))\right] d s,
$$

where $s \in[0,1]$ is the normalized length around the snake and to control the behavior of the snake naturally, $E_{\text {int }}$ is defined as follow:

$$
E_{\text {int }}=\frac{\alpha(s)\left|c^{\prime}(s)\right|^{2}+\beta(s)\left|c^{\prime \prime}(s)\right|^{2}}{2}
$$

where the first and the second derivatives of $c(s)$ are shown by $c^{\prime}(s)$ and $c^{\prime \prime}(s)$ with respect to $s$ and $\alpha$ and $\beta$ called elasticity and rigidity parameters, respectively, which are the weighting parameters of contour. The term of external energy, $E_{\text {ext }}$, attracts snake to the chosen low-level features (such as edge points) such that it has smaller value near the object boundary and bigger value in other areas. Using the calculation of variation, minimization of $E$ leads to the Euler-Lagrange equation as follow:

$$
\alpha c^{\prime \prime}(s)-\beta c^{\prime \prime \prime \prime}(s)-\nabla E_{\text {ext }}=0
$$

which can be regarded as a force balance equation:

$$
F_{\text {int }}+F_{\text {ext }}=0
$$

by considering $F_{\text {int }}=\alpha c^{\prime \prime}(s)-\beta c^{\prime \prime \prime \prime}(s)$, and $F_{\text {ext }}=-\nabla E_{\text {ext }}$. Internal force, $F_{\text {int }}$, discourages both stretching and bending and the external force, $F_{e x t}$, pulls the active contour to the desired image edges. Finally, the curve stops at the position with force balance. In order to solve (8), suppose that the active contour be a function of time $t$ (in addition to $s$ ), that is, $c(s, t)$ which satisfies the following equation:

$$
c_{t}(s, t)=\alpha c^{\prime \prime}(s)-\beta c^{\prime \prime \prime \prime}(s)-\nabla E_{\text {ext }} .
$$

Then the solution of (8) becomes from (10). The term of $E_{\text {ext }}$ can be computed with a very simple energy functional for the original image $I_{0}(x, y)$ as follow:

$$
E_{\text {ext }}=-\left|\nabla I_{0}(x, y)\right|,
$$

Limit capture range and poor convergence to the concave regions are two major issues in ACM. Fig. 3 shows an example of concave regions which has not been captured. 


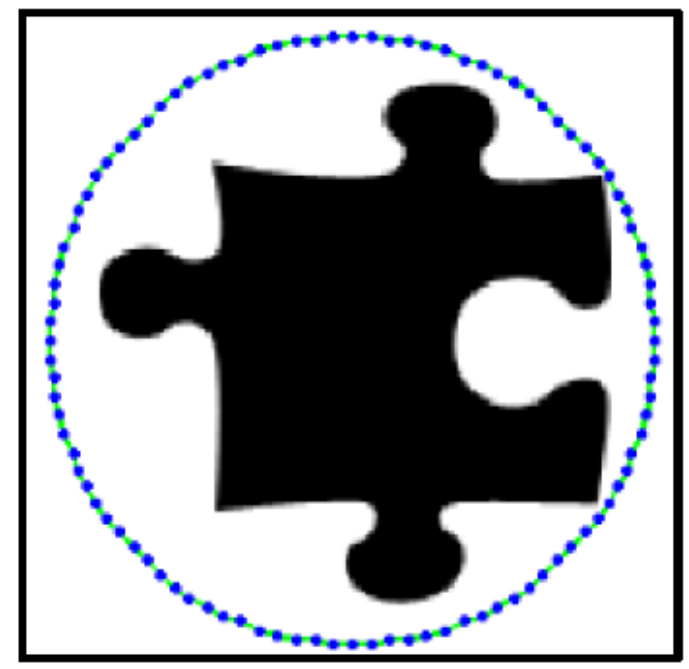

(a)

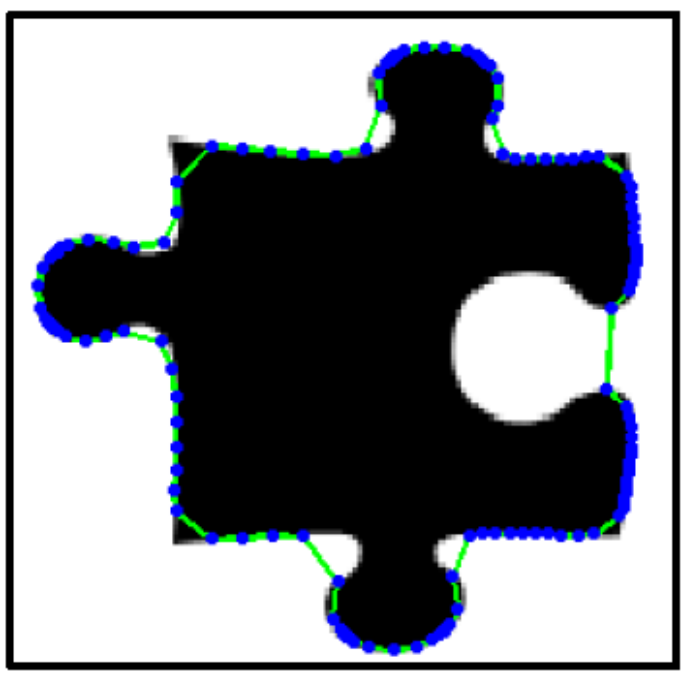

(b)

Figure 3. An example of concave regions; (a) is the initial snake, and (b) is the final state of snake (by Kass) occurred after 2346 iterations

\subsection{Geometric active contours (GAC) models}

One of the most popular level set representations with edge-based models is the Geometric active contours (GAC) model ${ }^{16}$. This model utilizes image gradient to construct an ESF. Usually, a positive, decreasing and regular ESF like $g(t)$ is used such that $\lim _{t \rightarrow \infty} g(t)=0$. For instance $g(|\nabla I|)$ can be computed using the following formula:

$$
g(|\nabla I|)=\frac{1}{1+\left|\nabla G_{\sigma} * I\right|^{2}}
$$

where $G_{\sigma} * I$ shows the convolution of Gaussian kernel (standard deviation of $\sigma$ ) and image $I$. However, for digital images the discrete gradients are bounded and therefore, $(12)$ will never be zero on edges. To overcome this issue, given $\Omega$ be a bounded open subset of $\mathbb{R}^{2}$ and $I:[0, a] \times[0, b] \rightarrow \mathbb{R}^{+}$be a given image. consider $c(q):[0,1] \rightarrow \mathbb{R}^{2}$ be a parameterized planar curve in $\Omega$. The GAC model is formulated with a minimization of the following energy functional:

$$
E_{G A C}(C)=\int_{0}^{1} g\left(|(\nabla I(C(q)) \mid)| C^{\prime}(q) \mid d q\right.
$$

where $\mathrm{g}$ is computed by (12). Using calculation of variation we could get the Euler-Lagrange equation of (13) as follow:

$$
C_{t}=g(|\nabla I|) \kappa \vec{N}-(\nabla g \cdot \vec{N}) \vec{N}
$$

where the curvature of the contour and inward normal vector to the curve are shown with $\kappa$ and $\vec{N}$, respectively. Also, in order to increase the propagation speed, term $\alpha$ is added as a constant velocity as follow:

$$
C_{t}=g(|\nabla I|)(\kappa+\alpha) \vec{N}-(\nabla g \cdot \vec{N}) \vec{N} .
$$

Level set function for the GAC model is:

$$
\frac{\partial \phi}{\partial t}=g(|\nabla \phi|)\left(\operatorname{div}\left(\frac{\nabla \phi}{|\nabla \phi|}\right)+\alpha\right)+\nabla g . \nabla \phi
$$

where the level set $\phi$ is approximated by $\nabla \phi$ and to control the contour shrinking or expanding, the balloon force $\alpha$ is used. The GAC model suffers some major disadvantages as follow: 
- High computational cost due to computation of gradient of the curvature approximation of the current level set $\phi(\nabla \phi)$ at each iteration.

- Since the GAC model is in terms of the curvature and the gradient, only local boundary information is used. These lead the GAC model to be affected by input noise. This problem is shown in Fig. 4.

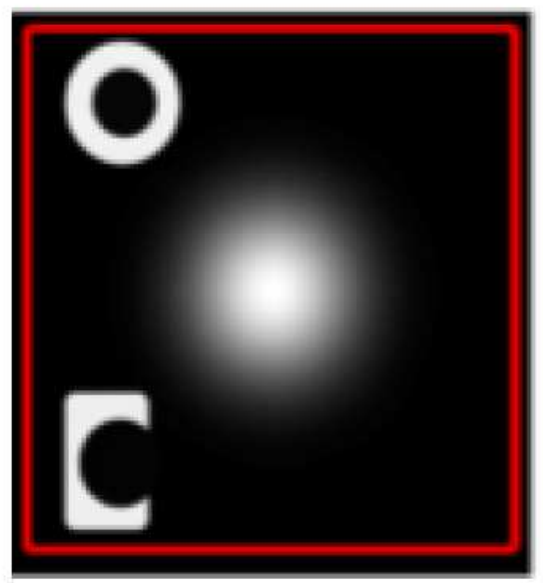

(a)

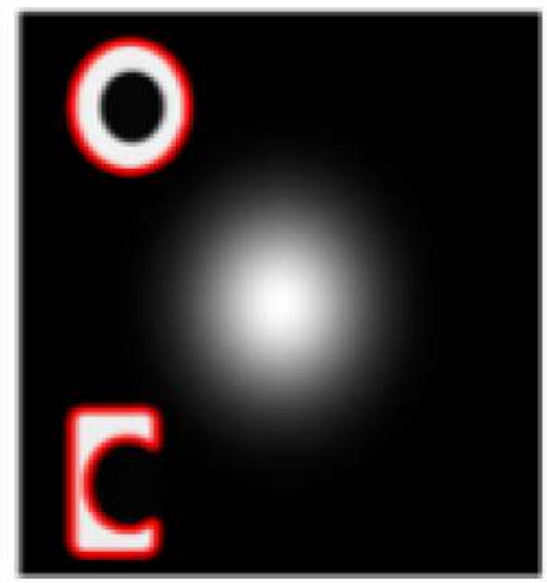

(b)

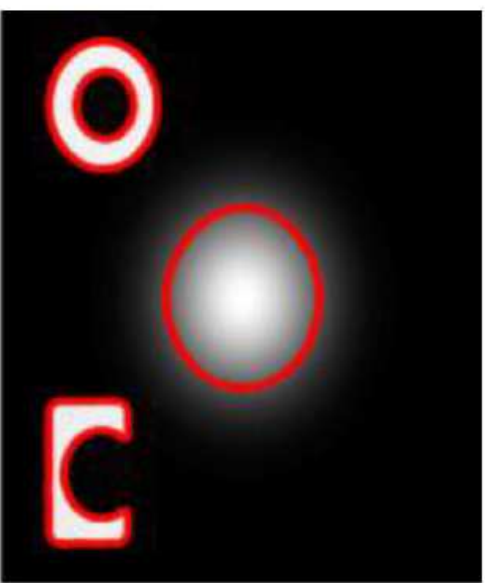

(c)

Figure 4. Segmentation result on a synthetic image based on GAC model; (a) the initial contour shown in red color, (b) the final segmentation result, and (c) shows all of the boundaries that should be surrounded

\subsection{The C-V model}

The $\mathrm{C}-\mathrm{V}$ model is a parametric representation with region-based description presented by Chan and Vese ${ }^{18}$. For a given image $I$ in domain $\Omega$, the $\mathrm{C}-\mathrm{V}$ model is formulated (parametric) by minimizing the following energy functional:

$$
\begin{aligned}
E_{C-V}= & \lambda_{1} \int_{\text {inside }(c)}\left|I(x)-c_{1}\right|^{2} d x \\
& +\lambda_{2} \int_{\text {outside }(C)}\left|I(x)-c_{2}\right|^{2} d x, \quad x \in \Omega
\end{aligned}
$$

where $c_{1}$ and $c_{2}$ show the average intensities inside and outside the contour, respectively. Also, with the level set method, it is assumed that:

$$
\left\{\begin{array}{c}
C=\{x \in \varphi: \phi(x)=0\} \\
\text { inside }(C)=\{x \in \varphi: \phi(x)>0\} \\
\text { outside }(C)=\{x \in \varphi: \phi(x)<0\}
\end{array}\right.
$$

where $C$ and $\phi(x)$ show level set and active curve in the image, respectively. By minimizing (12), $c_{1}$ and $c_{2}$ are computed as follow:

$$
\begin{aligned}
& c_{1}(\phi)=\frac{\int_{\Omega} I(x) \cdot H(x) d x}{\int_{\Omega} H(x) d x}, \\
& c_{2}(\phi)=\frac{\int_{\Omega} I(x) \cdot(1-H(x)) d x}{\int_{\Omega}(1-H(x)) d x},
\end{aligned}
$$

where $H(x)$ is the Heaviside function. The regularized versions of these functions are as follow:

$$
H_{\varepsilon}^{(z)}=\frac{1}{2}\left(1+\frac{2}{\pi} \arctan \left(\frac{z}{\varepsilon}\right)\right)
$$




$$
c_{2}(\phi)=\frac{\int_{\Omega} I(x) \cdot(1-H(x)) d x}{\int_{\Omega}(1-H(x)) d x},
$$

The zero level set $(\phi(x)=0)$ is updated by calculating the corresponding variation level set:

$$
\frac{\partial \phi}{\partial t}=\delta(\phi)\left[\mu \nabla\left(\frac{\nabla \phi}{|\nabla \phi|}\right)-v-\lambda_{1}\left(I-c_{1}\right)^{2}+\lambda_{2}\left(I-c_{2}\right)^{2}\right],
$$

where the smoothness of the level set is controlled by $\mu \geq 0$, two parameters the image data-driven the force inside and outside the contour, respectively. $v \geq 0$ increases the propagation speed. Two major disadvantages of $\mathrm{C}-\mathrm{V}$ model are as follow:

- Similar to GAC model, C-V model also needs to calculate the curvature approximation $\delta(\phi)$, which needs to high computational expense.

- Despite power of global segmentation of $\mathrm{C}-\mathrm{V}$ model with a proper initial contour, it cannot extract the interior contour without setting the initial inside the object and fails to extract all the objects. This problem has been shown in Fig. 5.

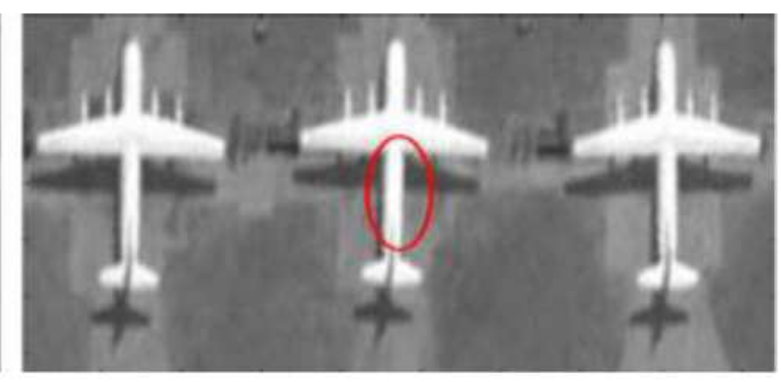

(a)

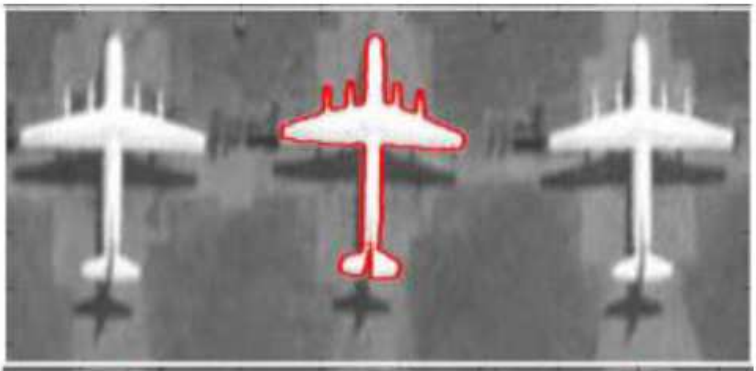

(b)

Figure 5. An example of global segmentation property of C-V model. (a) shows the initial contour, and (b) shows the segmentation result of $\mathrm{CV}$ method which can not extract all boundaries in the image

\subsection{Deep learning approaches}

Deep learning models demonstrated improved results, however they are limited to the pixel-wise fitting of the segmentation map. This limitation can be tackled by taking into account the size of boundaries and the areas inside and outside the region of interest during the learning process. This can be achieved by using an active contour loss function inspired by ACMs. This new loss function combines geometrical information with region similarity hence it provides more precise segmentation. The ACM loss function is used as a loss function in many deep learning models as in ${ }^{42-45}$.

\section{The state-of-the-art methods}

In this section, some of best proposed ACMs are reviewed and major advantages and disadvantages are presented.

\subsection{Magnetostatic active contour (MAC) model}

The magnetostatic active contour (MAC) model is based on the level set representation with edge-based description ${ }^{17}$. The advantages of MAC compared to other related works are as follow:

- Significant improvement in initialization invariance.

- Significant improvement in convergence capability; the contour attracts into deep concave regions.

- Is not affected by stationary point and saddle point problems.

- It is able to capture complex geometries. 
- It is able to capture multiple objects with a single initial contour.

MAC represents active contour using an implicit model in which the contour, $c$, is defined as follow:

$$
c=\{\bar{x} \mid \phi(\bar{x})=0\},
$$

where $\phi: \mathbb{R}^{2} \rightarrow \mathbb{R}$. The motion of contour to a partial differential equation (PDE) on the contour is related by MAC, as follow:

$$
\frac{\partial \phi}{\partial t}=-\nabla \phi \bar{v}(t)
$$

where $\bar{v}(t)$ obtains the velocity of the contour movement. For image segmentation MAC considers the following PDE:

$$
\frac{\partial \phi}{\partial t}=\alpha s(\bar{x}) \nabla\left(\frac{\nabla \phi}{|\nabla \phi|}\right)|\nabla \phi|-(1-\alpha) F(\bar{x}) \nabla \phi,
$$

where $\alpha, s(\bar{x})$, and $F(\bar{x})$ are a real constant, the stopping function (Sobel filter), and the magnetostatic force, respectively.

\subsection{Online region-based active contour model (ORACM)}

The ORACM is introduced as representing the level set with region-based ACM. The model do not require any parameter. Compared to traditional ACMs, the ORACM requires less time without a change in the segmentation accuracy. In each iteration, the ORCAM performs block thresholding. Rigorous boundaries and small particles that do not belong to the object are produced out of this process. To tackle this problem, morphological operations like closing and opening are applied. The level set function, $\phi(x)$, is initialized to constants, which have different signs such as -1 and +1 inside and outside the contour. A simple and efficient level set updating formulation is used in ORACM as follow:

$$
\frac{\partial \phi}{\partial t}=H(\operatorname{SPF}(I(x))) \cdot \phi(x),
$$

where $H($.$) is the Heaviside function, and \operatorname{SPF}($.$) is the signed pressure function defined as follow:$

$$
\operatorname{SPF}(I(x, y))=\frac{I(x, y)-\frac{c_{1}-c_{2}}{2}}{\max \left(\left|I(x, y)-\frac{c_{1}-c_{2}}{2}\right|\right)},
$$

where $I$ and $\phi$ show an input image and the current level set, respectively. Two parameters $c_{1}$ and $c_{2}$ have the same definition to (20) and (21), respectively. The advantages of ORACM are as follow:

- Decreasing efficiency without changing the accuracy of the image segmentation process.

- Accurate segmentation of all object regions in both inside and outside for medical, real, and synthetic images with holes, complex background, weak edges, and high noise.

The disadvantage of ORACM is that it supports only bimodal segmentation of piecewise constant intensity distribution. Therefore, the application of ORACM is limited to the cases satisfied the above constraints. It should be noted that two types of ORACM have been conducted by researchers called ORACM1 and ORACM2 which consider ORACM without or with the morphological operations, respectively.

\subsection{Selective binary and gaussian filtering regularized level set (SBGFRLS)}

The SBGFRLS ${ }^{25}$ is an ACM based on level set representation with region-based description. This model uses both global and local information. At the initialization step a user-defined active contour is determined and it is continuously updated by a region-based signed pressure function (SPF) defined by (25). Unlike $\mathrm{C}-\mathrm{V}$ method in obtaining $c_{1}$ and $c_{2}$ using (20) and (21), SBGFRLS uses the $H$ Heaviside function described in (22) only with $\varepsilon=0$. The SPF function tunes the signs of the pressure force inside and outside the region of interest. For example, the contour shrinks and expands based on whether its outside or inside the object. The SBGFRLS obtains the corresponding variation level set by:

$$
\frac{\partial \phi}{\partial t}=\operatorname{SPF}(I(x)) \cdot \alpha \cdot|\nabla \phi|,
$$

where to control the speed, constant $\alpha$ is used. The advantages of SBGFRLS are as follow:

- Powerful for noise because the image statistical information is used to stop the curve evolution on the desired boundaries. 
- Well performance in images with weak edges or even without edges.

- Initial curve can be defined anywhere to extract the interior boundaries of the objects

However, the disadvantages of the ACM with SBGFRLS include the following cases:

- Difficult to use ACM with SBGFRLS on different images because it needs to be tuned according to the image and that's why it can't be used on real-time video images.

- The slowness of the method caused by propagating the SPF function results in the boundary of the level set function only using $|\nabla \phi|$ at the level set. Updating only the boundary of the level set is the main cause of the slowness.

\subsection{Level set active contour model (LSACM)}

The LSACM is a level set method relies on the region for image segmentation in the presence of intensity inhomogeneity. This method models the inhomogeneous objects as Gaussian distributions of different means and variances. Using a sliding window, the original image is mapped into another domain.In this area, every object's intensity distribution is still Gaussian but more distinct. It is possible to approximate the means of the Gaussian distributions in the transformed domain by multiplying a bias field with the original signal inside the window. A functional energy of maximum likelihood is then defined in the entire image region, which combines the bias field, the level set function, and the partly constant function which approximates the true image signal ${ }^{8}$.

The method works by combining information from neighbouring pixels that belong to the same class. In this approach, the desired object is separated from its background. Given $N R_{x}$ a neighboring region centered at location $x$, i.e., $N R_{x}=\{y|| y-x \mid \leq$ $\rho$ \}, where for the region $N R_{x}, \rho$ is the radius of that. The whole image domain $\Omega$ can be represented as $\Omega=\cup_{i=1, \ldots, n} \Omega_{i}$ with $\Omega_{i} \cap \Omega_{i}=\emptyset$, for all $i \neq j$, where $\Omega_{i}$ is the ith object region. A mapping $\mathscr{T}: I\left(x \mid \theta_{i}, B\right) \rightarrow \mathscr{I}\left(x \mid \theta_{i}, B\right)$ from original image intensity domain $I D(\mathscr{T})$ to another domain $R D(\mathscr{T})$ by averaging image intensities is defined.

$$
\mathscr{I}\left(x \mid \theta_{i}, B\right)=\frac{1}{L_{i}(x)} \int_{\Omega_{i} \cap N R_{x}} I\left(y \mid \theta_{i}, B, x\right) d y
$$

where the number of pixels in region $\Omega_{i} \cap N R_{x}$ obtains with $L_{i}(x)=\left|\Omega_{i} \cap N R_{x}\right|$. The intensity of pixel $x$ is assumed to be independently distributed with a Gaussian distribution, that is, $P\left(\mathscr{I}\left(x \mid \theta_{i}, B\right)\right)=\mathscr{N}\left(I \mid U_{i}(x), \frac{\sigma_{i}^{2}}{L_{i}(x)}\right)$ where $U_{i}$ and $\sigma_{i}$ are the spatial varying mean and the standard deviation subject to the object in region $\Omega_{i}$, for all $\mathscr{I}\left(x \mid \theta_{i}\right) \in R D(\mathscr{I})$.

The advantages of this method are: it achieves soft classification, it is robust against noise and it mitigates over smoothing object boundaries problem.

The disadvantages are: only the neighbouring intensities belonging to the same class contribute to each class and the overlapping parts of the statistical distributions among different classes of intensities are suppressed.

\subsection{Region-scalable fitting and optimized Laplacian of Gaussian energy (RSFOLGE)}

The RSFOLGE applies a LoG energy term optimized by an energy functional. The advantage is smoothing the homogeneous regions while enhancing edge information at the same time. Then it integrates the optimized LoG energy term with the region-scalable fitting energy term. The advantage is making use of of local region information to drive the curve towards the boundaries. The advantage of the RSFOLGE model is that it achieves accurate image segmentation and it is insensitive to the positions of initial contour. ${ }^{9}$. The energy functional used to optimize the LoG of the image is as follow:

$$
\begin{aligned}
E^{L Q G}(L)= & \iint_{\Omega} g([\nabla I]) \times(L-0)^{2}+(1-g(|\nabla I|)) \\
& \times\left(L-\beta \times \Delta\left(G_{\sigma} * I\right)\right)^{2} d x d y
\end{aligned}
$$

where the value of optimized LoG of the image is represented with $L$, and $g(\nabla I)=e^{-a \mid \nabla G_{o} * I}, a, \beta$ are positive constants. A edge indicator is defined by $g(\nabla I)$. The values of $g(\nabla I)$ are small and approximately equal to 0 at the locations near the object boundaries. $\Delta\left(G_{\sigma} * I\right)$ is:

$$
\Delta\left(G_{\sigma} * I\right)=\left[\frac{\partial^{2} G_{\sigma}(x, y)}{\partial x^{2}}+\frac{\partial^{2} G_{\sigma}(x, y)}{\partial y^{2}}\right] * I(x, y)
$$

where $G_{\sigma}(x, y)$ is a Gaussian kernel function with standard deviation $\sigma$.

The advantages of this method are that it develops the robustness of initialization and it has accurate segmentation results compared with the original region scalable fitting (RSF) method. Moreover, the optimization process is applied to the edge stopping function which used in edge-based ACMs. 
The disadvantage of this method is that it fails when objects inside image have significantly different intensity values which can be tackled by using multi-phase segmentation.

A simple and universal method of improving the robustness of initial contour for these local fitting-based models is

presented $^{10}$. The core idea of proposed method is exchanging the fitting values on the two sides of contour, so that the fitting values inside the contour are always larger (or smaller) than the values outside the contour in the process of curve evolution. In this way, the whole curve will evolve along the inner (or outer) boundaries of object, and less likely to be stuck in the object or background.

Advantage: enhance the robustness of initial contour and keep the original LRF.

\subsection{Adaptive local-fitting (ALF) method}

The ALF model pushes the initial contour towards object boundary using adaptive local fitting energy and regularization energy. This method shows an accurate way to separate the region of interest. The traditional methods assume the intensities in the local region are constant, however, the LBF method finds an optimal solution by fitting the original image using an adaptive technique. $^{46}$

The ALF method improves on the Local Binary Fitting method. The energy function of the ALF is:

$$
\begin{aligned}
E^{A L F}= & \int \sum_{i=1}^{N}\left(\kappa_{i} \int w^{x}(x-y)\left|I(y)-\mu_{i}(x)-\lambda(x) \bar{\sigma}(y)\right|^{2}\right. \\
& \left.\times M_{i}(\Phi(y)) d y\right) d x \\
& +\beta \int \frac{1}{2}(|\nabla \Phi(x)|-1)^{2} d x+v \int \delta(\Phi(x))|\nabla \Phi(x)| d x
\end{aligned}
$$

where $w^{x}$ is a truncated weight function, $\bar{\sigma}$ is a appropriate estimation to $\sigma$ and $M_{i}(\Phi(y))$ is a membership function satisfying:

$$
M_{i}(\Phi(y))=\left\{\begin{array}{l}
1, y \in \Omega_{i} \\
0, \text { else }
\end{array}\right.
$$

In Eq. (27), when $\lambda(x)=0$ or $\bar{\sigma}(y)=0$, it is the same as LBF model. So, LBF model can be seen as special case of the proposed ALF model. Nevertheless, LBF discards the local information about intensity variance Despite the necessary for improving efficiency of the method, the model can extract more details and performs robust to intensity inhomogeneity and noises.

\subsection{Fuzzy Region-Based active contour model (FRBACM)}

The FRBACM is driven by weighting global and local fitting energy, wherein fuzzy region energy with local spatial image information is proposed. The segmentation results of this method are independent of initialization. To extract object boundaries while maintaining its distance, an initial evolving curve of pseudo level set function (LSF) followed by the pseudo-LSF and further smoothed by edge energy is proposed. This method consists of fuzzy region energy which is formulated with local spatial image information and edge energy which use the evolving curve to stop the object boundaries. The fuzzy region energy is strictly convex and used to drive the motion of the evolving curves. To minimize the energy functional, the fuzzy region energy directly calculate the change of the fuzzy region energy instead of using the Euler-Lagrange equation ${ }^{47}$. The fuzzy energy obtains: 


$$
\begin{aligned}
\Delta F(x)= & g t_{1}\left(\alpha \frac{\omega(x, y) * \Delta u_{1}(x)}{\omega(x, y) *\left(t_{1}+\Delta u_{1}(x)\right)}\left(I(x)-f_{o}\right)\right. \\
& \left.+\beta \frac{\Delta u_{1}}{t_{1}+\Delta u_{1}}\left(I(x)-c_{1}\right)\right)^{2} \\
& +g t_{2}\left(\alpha \frac{\omega(x, y) * \Delta u_{2}(x)}{\omega(x, y) *\left(t_{2}+\Delta u_{2}(x)\right)}\left(I(x)-f_{b}\right)\right. \\
& \left.+\beta \frac{\Delta u_{2}}{t_{2}+\Delta u_{2}}\left(I(x)-c_{2}\right)\right)^{2} \\
& +g \Delta u_{1}(x)\left(\frac{\alpha \omega(x, y) * t_{1}}{\omega(x, y) *\left(t_{1}+\Delta u_{1}(x)\right)}\left(I(x)-f_{o}\right)\right. \\
& \left.+\frac{\beta t_{1}}{t_{1}+\Delta u_{1}(x)}\left(I(x)-c_{1}\right)\right)^{2} \\
& +g \Delta u_{2}(x)\left(\frac{\alpha \omega(x, y) * t_{2}}{\omega(x, y) *\left(t_{2}+\Delta u_{2}(x)\right)}\left(I(x)-f_{b}\right)\right. \\
& \left.+\frac{\beta t_{2}}{t_{2}}+\Delta u_{2}(x)\left(I(x)-c_{2}\right)\right)^{2}
\end{aligned}
$$

weighting constants $\alpha, \beta$ and the edge detector matrix $g$. This proposed model succeed in extracting objects in different situations such as images with noise, images with intensity inhomogeneity, however, this model fails when the similarity between the object and its background is high.

\subsection{Global and Local Signed Energy-Based Pressure Force(GLSEPF)}

The GLSEPF uses the energy difference between the inner and outer energies to obtain the contour of the object. This model will improve the initial curve robustness. The local signed energy-based pressure force (LSEPF) is determined by means of the pixel-by-pixel energy difference within the local neighborhood region. This LSEPF can handle images with depth inhomogeneity and noise. Global image information and local energy information are used respectively for global and local force propagation functions. Global and local variances are used to balance the weights of GSEPF and LSEPF automatically, which can solve the problem of parameter setting. In the meantime, a regularization term and a penalty term are added in order to prevent re-initialization during iterations and smooth the level set function ${ }^{48}$. The level set formulation of the GSEPF model is written as:

$$
\text { GSEPF model: } \begin{aligned}
\frac{\partial \phi}{\partial t}= & \frac{\Delta E^{g}(I(x))}{\max \left(\left|\Delta E^{g}(I(x))\right|\right)} \cdot\left|c_{1}-c_{2}\right| \cdot \nabla \phi \\
& +\mu \delta(\phi) \cdot \operatorname{div}\left(\frac{\nabla \phi}{|\nabla \phi|}\right) \\
& +v\left(\nabla^{2} \phi-\operatorname{div}\left(\frac{\nabla \phi}{|\nabla \phi|}\right)\right)
\end{aligned}
$$

where $\Delta E^{g}(I(x))$ is the global energy. Objects in images with noise and intensity can be detected by incorporation of the global and local image information, however, this for color images, the segmentation is poor because only the intensity information is used.

\section{Evaluation}

This section presents the experimental results from the active contour methods that defined in previous section for comparison purposes. We also explain the implementation of the methods.

\subsection{Database}

The pneumonia infection due to COVID-19 is generally appraised in hospitals using the chest X-ray/CTI ${ }^{40}$. The visibility of CTI is better compared to chest X-ray and hence, this research considers the CTI for the experimental investigation. The COVID-19 is a novel disease and the availability of the images for the research is very limited due to various constraints. This work considered the COVID-19 pneumonia image database of ${ }^{26}$ to test the performance of the proposed image processing 
method. This database consists of 100 CTI with dimensions $512 \times 512 \times 1$ pixels and all the existing images are associated with related Ground-Truth-Images (GTI). Th overall distribution of patients is provided in Table 1. Also, figure 6 shows samples of Covid-19 CT image and their ground truth.

Table 1. The overall distribution of samples used in the database.

\begin{tabular}{ll}
\hline Range of ages of patients & $32-86$ (years) \\
No. of total patients (men+women+NA) & $49(=27+11+11)$ \\
Minimum no. of Covid-19 CT images per patient & 1 \\
Maximum no. of Covid-19 CT images per patient & 13 \\
Total no. of Covid-19 CT images & 100 \\
\hline
\end{tabular}

\subsection{Lung region extraction}

In this work, we first remove the artifacts such as bones and other body segments from the test image using a thresholding filter in ${ }^{40}$. This thresholding filter separates the testing image into two sections based on a manually selected threshold. The selection of the threshold is done by trial and error analysis.

chosen threshold then works well on all the images considered in the proposed study. The considered images can be exactly separated based on its pixels. In addition, when the method is failed, we separated the part of images manually. The results attained with the threshold filter are depicted in Figure 7.

\subsection{Comparison the active contour methods}

In this section, the performances of the mentioned methods in the previous sections have been compared. We performed all simulations in MATLAB R2018a. All experiments were run on a 64-bit operating system with a CPU E5-2690 v3 @ 2.60 $\mathrm{GHz}, 64.0 \mathrm{~GB}$ of RAM, and a single NVIDIA GTX TITAN X. In order to implement the methods, their codes

have been provided by authors; The methods: C-V, SBGFRLS, and ORACM ${ }^{1}$, LSACM ${ }^{2}, \mathrm{RSFOLGE}^{3}, \mathrm{ALF}^{4}, \mathrm{FRAGL}^{5}$, GLSEPF $^{6}$ have been implemented in MATLAB code and the method MAC ${ }^{7}$ has been implemented in JAVA code. For all of the methods used on the database, the default parameters mentioned in their codes were retained. The experimental outcome confirms that the proposed methods provides the mean value of infection rate for further evaluation. Comparative analysis has been performed in the case of CT COVID-19 images. In order to test the robustness to the initialization, three initial contours including: inside, outside, and overlapping (cross) the target object have been considered. However, some methods failed the initial contours as shown in Table 2 .

As shown in this Table 2, in two cases: both methods MAC, ORACM can perform final segmentation by the three initial contours. Also, LSACM is failed only in one of the initial contour and rest of the methods are succeed only in one of the initial contour.

\subsection{Result}

To assess the methods, we utilized seven evaluation metrics: Dice, Jaccard, Bfscore, Precision, Recall, Iteration and time. Subsection II.C includes a detailed description of the measures. Furthermore, the quality of the methods results is shown in Figure 7. The quantitative results, in terms of the measures, from the database are shown in Tables 3 . According to the

\footnotetext{
${ }^{1}$ Available at http://iys.inonu.edu.tr /webpanel/dosyalar/1348/file/OnlineSeg.rar

${ }^{2}$ Available at http://www.comp.polyu.edu.hk/ cslzhang/LSACM/ LSACM.htm

${ }^{3}$ Available at https://github.com/dingkeyan93/Active-Contour-Model-Matlab-Code-Set

${ }^{4}$ Available at https://github.com/madd2014/ALF

${ }^{5}$ Available at https://github.com/fangchj2002/FRAGL

${ }^{6}$ Available at https://github.com/HuaxiangLiu/GLSEPF/

${ }^{7}$ Available at http://csvision.swan.ac.uk/public_html_XX/snakes/mac/index.html
}

Table 2. Success of active contour methods in obtaining infection area in terms of initial contour

\begin{tabular}{|cccccccccc|}
\hline Initial contour & C-V & SBGFRLS & MAC & ORACM & LSACM & RSFOLGE & ALF & FRAGL & GLSEPF \\
\hline Inside & Failed & Failed & Done & Done & Failed & Failed & Failed & Failed & Failed \\
\hline Outside & Failed & Failed & Done & Done & Failed & Done & Done & Failed & Failed \\
\hline Cross & Done & Failed & Done & Done & Done & Done & Failed & Done & Done \\
\hline
\end{tabular}



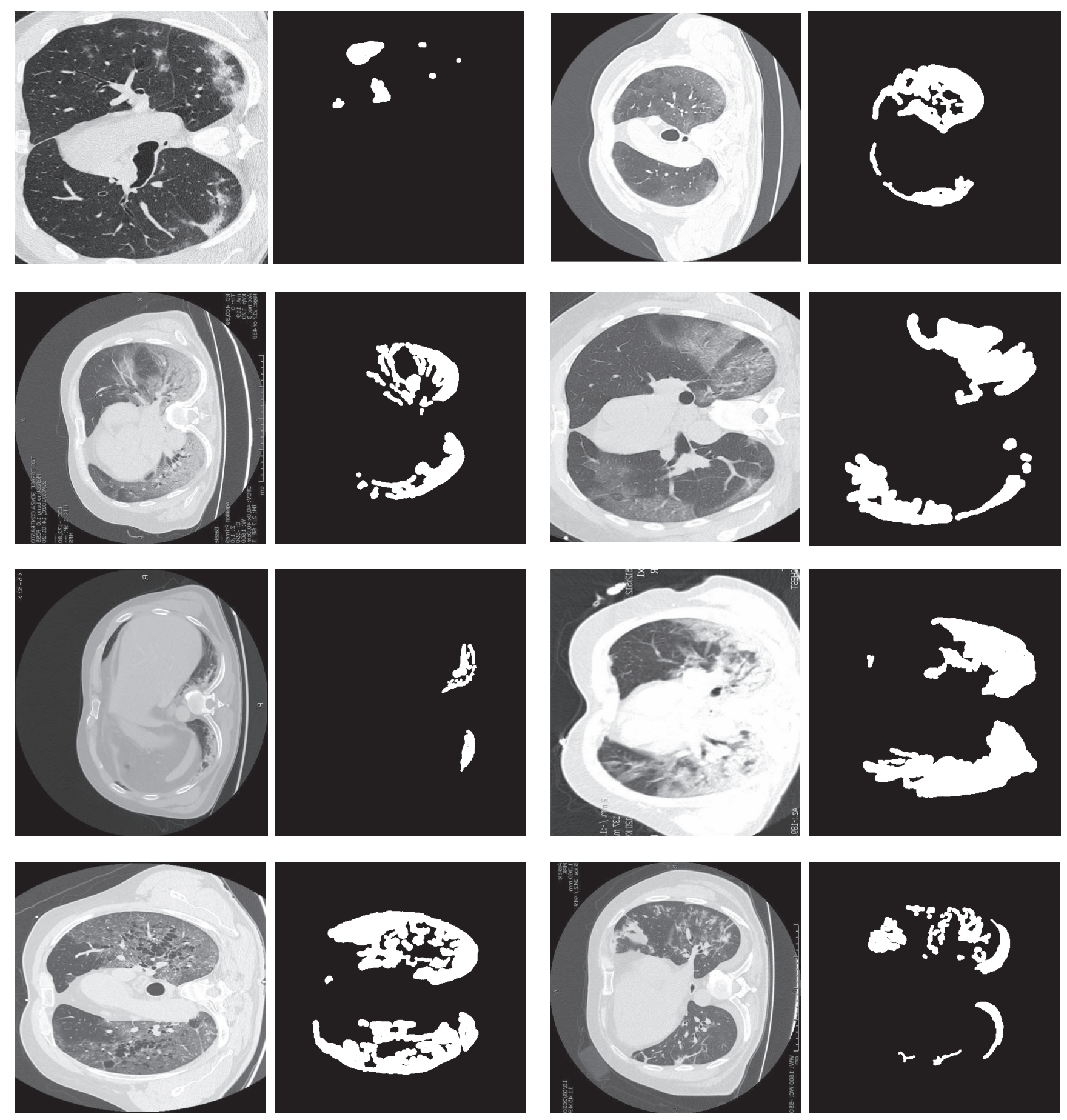

Figure 6. Samples of images of COVID-19 database and their ground truth.

evaluations, quantitative and qualitative results in segmentation obtain promising results, but the results should help researchers to improve the results in the sensitive application. Figure 7 presents six the chosen images from the database and outputs obtained by the methods. It should be noted that some methods are failed in at least one of the initial contour, therefore, we report best results of each the methods. In an overall evaluation, we can see that ORACM obtains the best results compared with other methods in both quantitative and qualitative evaluations. 
Table 3. Detailed evaluation of seven metrics based on Covid-19 CT images

\begin{tabular}{|ccccccccc|}
\hline Measure & C-V & MAC & ORACM & LSACM & RSFOLGE & ALF & FRAGL & GLSEPF \\
\hline Dice (\%) & 93.55 & 95.94 & 96.30 & 95.77 & 89.88 & 92.12 & $\mathbf{9 6 . 4 4}$ & 95.60 \\
\hline Jaccard (\%) & 88.31 & 92.32 & 93.06 & 92.01 & 82.49 & 85.70 & $\mathbf{9 3 . 2 1}$ & 91.77 \\
\hline Bfscore (\%) & 66.82 & 61.40 & $\mathbf{7 4 . 1 3}$ & 60.46 & 63.50 & 57.05 & 65.55 & 71.96 \\
\hline Precision (\%) & 84.44 & 92.37 & 77.73 & $\mathbf{9 6 . 8 9}$ & 73.03 & 93.33 & 91.33 & 68.24 \\
\hline Recall (\%) & 58.15 & 48.25 & 72.41 & 45.67 & 61.44 & 43.34 & 53.18 & $\mathbf{7 8 . 8 1}$ \\
\hline Iteration & 158 & 8500 & $\mathbf{5}$ & 200 & 250 & 8 & 10 & 30 \\
\hline Time (s) & 55 & 700 & $\mathbf{1 . 4}$ & 12 & 41.40 & 100 & 1.8 & 5.5 \\
\hline
\end{tabular}
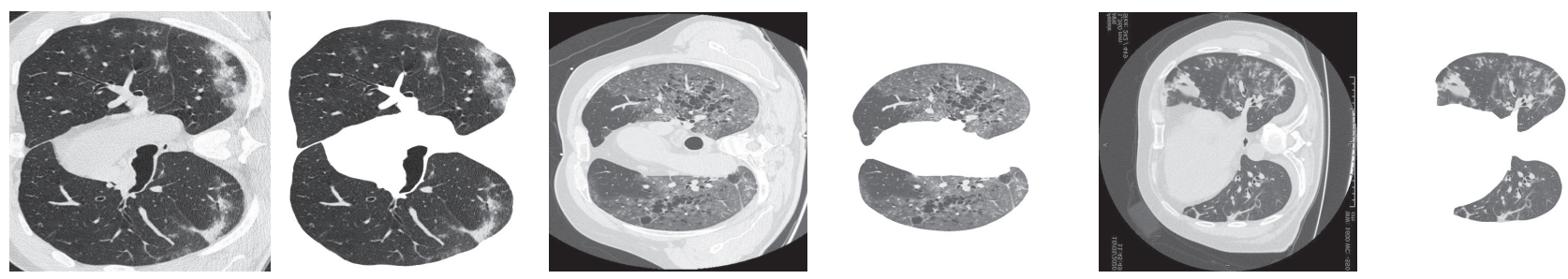

Figure 7. Outcomes of extraction lung region extraction

\subsubsection{Quantitative evaluation}

The quantitative results in terms of Dice, Jaccard, Bfscore, Precision, Recall, Iteration and time measured from the database are shown in Tables 3. The table reveal that ORACM executes considerably better than all of other techniques in terms of Bfscore, iteration and time and is as sound as the FRAGL method with respect to Dice and Jaccard measures. Therefore, as shown in the table, FRAGL obtains the first rank in terms of DICE and Jaccard measures. Compared with other methods, LSACM obtains the highest score in terms of precision and follow by ALF and MAC methods that come in second and third after the method. While GLSEPF first-place with regards to the recall measure, ORACM obtains the second rank and other methods have without promising results in the measure. As noted from the low value of the time and iteration, the ORACM and ALF are also more faster than the others. Since the Bfscore measures how close the predicted boundary of an object matches the ground truth boundary, ORACM preserves

boundaries better than other methods. Further, Jaccard and Dice similarities of 100\% means that the segmentations in the two images are a perfect match, FRAGL and ORACM obtain the best matched images compared to other methods.

\subsubsection{Qualitative evaluation}

Certain binary results processed on six example images of the database CT image are shown in Figure 8. As shown by the results, the RSFOLGE, MAC and C-V methods achieve clean binary images. However, the result of ORACM, FRAGL and GLSEPF methods are more effective and can efficiently produce a higher visual quality from the input image with a dark shadow. In the qualitative assessment, as shown in the figure, ORACM is smooth in keeping the boundaries in the samples. As exhibited, the ORACM, FRAGL and GLSEPF methods are successful in separating the infection area, which serves as the foreground, from the background, but they also fail in preserving thin strokes. Nevertheless, the methods accomplishes the best visual quality for the samples of the coronal and axial-view database.

\section{Conclusion}

This study is introductory to the automatic examination of the COVID-19 infection using CTSI. Locating a collection of clinical-grade images is a difficult task because of the sudden emergence of the disease. In this work, Active Contour methods are applied to the 2D lung CT scan slices to automatically extract the infected sections. This research tested methods from 2008 to 2020 which are divided in four classes which belong to contour representation and object boundary description categories. Beside to the brief explanation of the methods, advantages and disadvantage of the methods have been explored, in this paper. Moreover, a comparison in terms of the computational cost and accuracy has been conducted between the methods. Based on the experimental results, ORACM2 (Online region-based active contour model with the morphological operations) has overally the best performance in both speed and accuracy.

In future work, we aim to test other segmentation methods in other categories such as deep learning and clustering. Also, we will propose to implement : (i) automatic detection and classification COVID-19 cases into mild, moderate and severe 


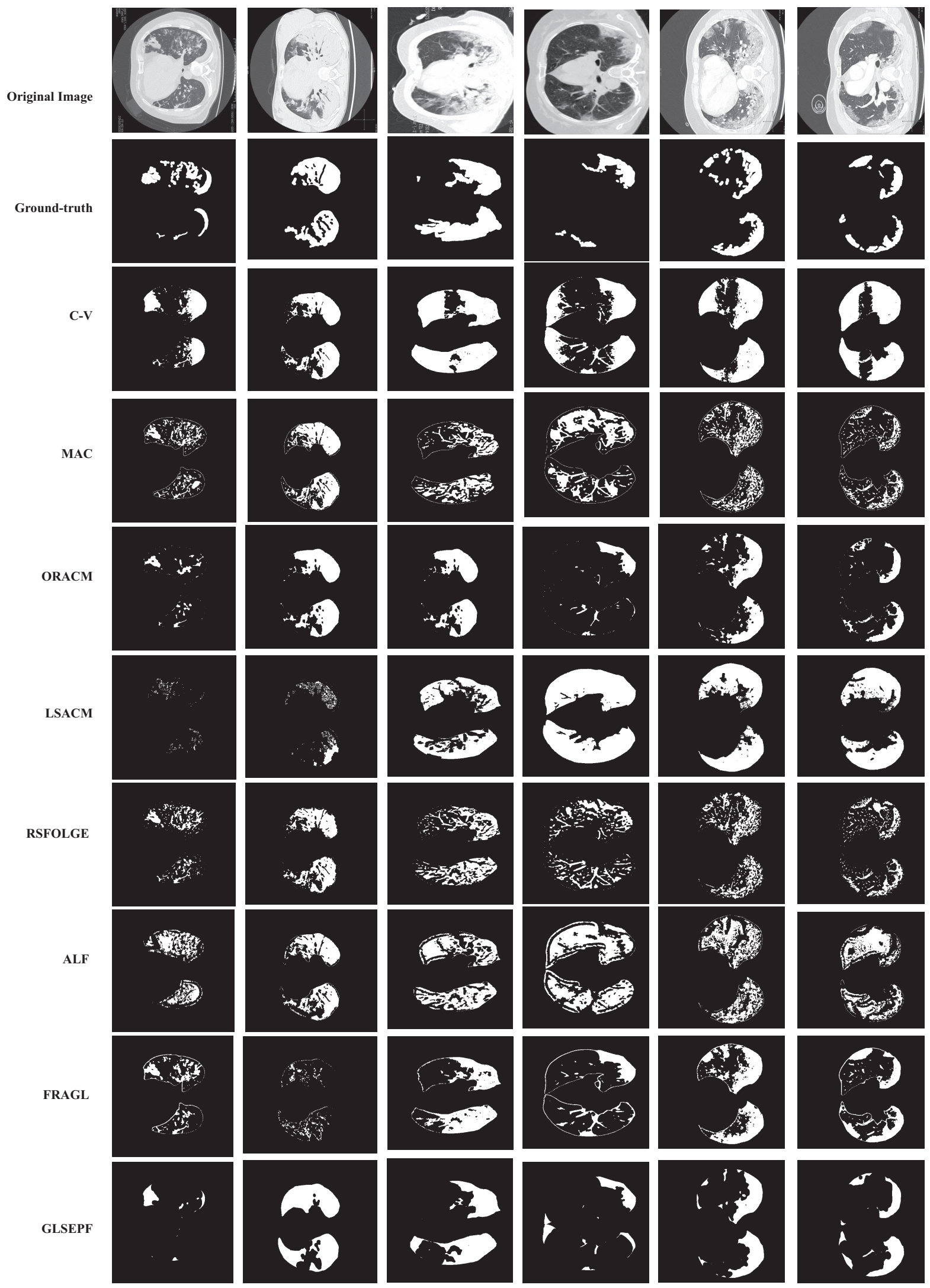

Figure 8. Outcomes of all active contour models in terms of the database. 
classes; (ii) automatic detection of the disease progress; and (iii) automatic classification of the CTSI slices into normal and COVID-19 pneumonia classes.

\section{Acknowledgments}

This publication was jointly supported by Qatar University ERG-XXX-XX-XX [GRANT CODE]. The findings achieved herein are solely the responsibility of the authors

\section{References}

1. Wu, Y.-H. et al. Jcs: An explainable covid-19 diagnosis system by joint classification and segmentation. arXiv preprint arXiv:2004.07054 (2020).

2. Ribbens, A., Hermans, J., Maes, F., Vandermeulen, D. \& Suetens, P. Unsupervised segmentation, clustering, and groupwise registration of heterogeneous populations of brain $\mathrm{mr}$ images. IEEE transactions on medical imaging 33, 201-224 (2013).

3. Gong, M., Liang, Y., Shi, J., Ma, W. \& Ma, J. Fuzzy c-means clustering with local information and kernel metric for image segmentation. IEEE transactions on image processing 22, 573-584 (2012).

4. Kuo, J.-w. et al. Nested graph cut for automatic segmentation of high-frequency ultrasound images of the mouse embryo. IEEE transactions on medical imaging 35, 427-441 (2015).

5. Li, G. et al. Automatic liver segmentation based on shape constraints and deformable graph cut in ct images. IEEE Transactions on Image Process. 24, 5315-5329 (2015).

6. He, K., Gkioxari, G., Dollár, P. \& Girshick, R. Mask r-cnn. In Proceedings of the IEEE international conference on computer vision, 2961-2969 (2017).

7. Ronneberger, O., Fischer, P. \& Brox, T. U-net: Convolutional networks for biomedical image segmentation. In International Conference on Medical image computing and computer-assisted intervention, 234-241 (Springer, 2015).

8. Zhang, K., Zhang, L., Lam, K.-M. \& Zhang, D. A level set approach to image segmentation with intensity inhomogeneity. IEEE transactions on cybernetics 46, 546-557 (2015).

9. Ding, K., Xiao, L. \& Weng, G. Active contours driven by region-scalable fitting and optimized laplacian of gaussian energy for image segmentation. Signal Process. 134, 224-233 (2017).

10. Ding, K. \& Xiao, L. A simple method to improve initialization robustness for active contours driven by local region fitting energy. arXiv preprint arXiv:1802.10437 (2018).

11. Kim, W. \& Kim, C. Active contours driven by the salient edge energy model. IEEE Transactions on Image Process. 22, 1667-1673 (2012).

12. Lecellier, F. et al. Region-based active contours with exponential family observations. J. Math. Imaging Vis. 36, 28 (2010).

13. Xu, T., Cheng, I. \& Mandal, M. An improved fluid vector flow for cavity segmentation in chest radiographs. In 201020 th International Conference on Pattern Recognition, 3376-3379 (IEEE, 2010).

14. Ronfard, R. Region-based strategies for active contour models. Int. journal computer vision 13, 229-251 (1994).

15. Huang, R., Pavlovic, V. \& Metaxas, D. N. A graphical model framework for coupling mrfs and deformable models. In Proceedings of the 2004 IEEE Computer Society Conference on Computer Vision and Pattern Recognition, 2004. CVPR 2004., vol. 2, II-II (IEEE, 2004).

16. Caselles, V., Kimmel, R. \& Sapiro, G. Geodesic active contours. Int. journal computer vision 22, 61-79 (1997).

17. Xie, X. \& Mirmehdi, M. Mac: Magnetostatic active contour model. IEEE Transactions on pattern analysis machine intelligence 30, 632-646 (2008).

18. Chan, T. F. \& Vese, L. A. Active contours without edges. IEEE Transactions on image processing 10, 266-277 (2001).

19. Talu, M. F. Oracm: Online region-based active contour model. Expert. Syst. with Appl. 40, 6233-6240 (2013).

20. Kass, M., Witkin, A. \& Terzopoulos, D. Snakes: Active contour models. Int. journal computer vision 1, 321-331 (1988).

21. Wang, T., Cheng, I., Basu, A. et al. Fluid vector flow and applications in brain tumor segmentation. IEEE transactions on biomedical engineering 56, 781-789 (2009).

22. Liu, B. et al. Probability density difference-based active contour for ultrasound image segmentation. Pattern Recognit. 43, 2028-2042 (2010). 
23. Cohen, L. D. \& Cohen, I. Finite-element methods for active contour models and balloons for 2-d and 3-d images. IEEE Transactions on Pattern Analysis machine intelligence 15, 1131-1147 (1993).

24. Sethian, J. A. \& Sethian, J. Level set methods: Evolving interfaces in geometry, fluid mechanics, computer vision, and materials science, vol. 1999 (Cambridge University Press Cambridge, 1996).

25. Zhang, K., Zhang, L., Song, H. \& Zhou, W. Active contours with selective local or global segmentation: A new formulation and level set method. Image Vis. computing 28, 668-676 (2010).

26. Jenssen, H. B. Covid-19 ct segmentation dataset. http: //medicalsegmentation.com/covid19/ (accessed 04 10, 2020).

27. Rodriguez-Morales, A. J. et al. Clinical, laboratory and imaging features of covid-19: A systematic review and metaanalysis. Travel. medicine infectious disease 101623 (2020).

28. Shi, F. et al. Review of artificial intelligence techniques in imaging data acquisition, segmentation and diagnosis for covid-19. arXiv preprint arXiv:2004.02731 (2020).

29. Zheng, C. et al. Deep learning-based detection for covid-19 from chest ct using weak label. medRxiv (2020).

30. Cao, Y. et al. Longitudinal assessment of covid-19 using a deep learning-based quantitative ct pipeline: Illustration of two cases. Radiol. Cardiothorac. Imaging 2, e200082 (2020).

31. Huang, L. et al. Serial quantitative chest ct assessment of covid-19: Deep-learning approach. Radiol. Cardiothorac. Imaging 2, e200075 (2020).

32. Qi, X. et al. Machine learning-based ct radiomics model for predicting hospital stay in patients with pneumonia associated with sars-cov-2 infection: A multicenter study. medRxiv (2020).

33. Gozes, O. et al. Rapid ai development cycle for the coronavirus (covid-19) pandemic: Initial results for automated detection \& patient monitoring using deep learning ct image analysis. arXiv preprint arXiv:2003.05037 (2020).

34. Li, L. et al. Artificial intelligence distinguishes covid-19 from community acquired pneumonia on chest ct. Radiology 200905 (2020).

35. Jin, S. et al. Ai-assisted ct imaging analysis for covid-19 screening: Building and deploying a medical ai system in four weeks. medRxiv (2020).

36. Shan+, F. et al. Lung infection quantification of covid-19 in ct images with deep learning. arXiv preprint arXiv:2003.04655 (2020).

37. Tang, L., Zhang, X., Wang, Y. \& Zeng, X. Severe covid-19 pneumonia: Assessing inflammation burden with volumerendered chest ct. Radiol. Cardiothorac. Imaging 2, e200044 (2020).

38. Shen, C. et al. Quantitative computed tomography analysis for stratifying the severity of coronavirus disease 2019. $J$. Pharm. Analysis (2020).

39. Rajinikanth, V. et al. Harmony-search and otsu based system for coronavirus disease (covid-19) detection using lung ct scan images. arXiv preprint arXiv:2004.03431 (2020).

40. Rajinikanth, V., Kadry, S., Thanaraj, K. P., Kamalanand, K. \& Seo, S. Firefly-algorithm supported scheme to detect covid-19 lesion in lung ct scan images using shannon entropy and markov-random-field. arXiv preprint arXiv:2004.09239 (2020).

41. Roerdink, J. B. \& Meijster, A. The watershed transform: Definitions, algorithms and parallelization strategies. Fundamenta informaticae 41, 187-228 (2000).

42. Chen, X. et al. Learning active contour models for medical image segmentation. In Proceedings of the IEEE Conference on Computer Vision and Pattern Recognition, 11632-11640 (2019).

43. Marcos, D. et al. Learning deep structured active contours end-to-end. In Proceedings of the IEEE Conference on Computer Vision and Pattern Recognition, 8877-8885 (2018).

44. Gur, S., Wolf, L., Golgher, L. \& Blinder, P. Unsupervised microvascular image segmentation using an active contours mimicking neural network. In Proceedings of the IEEE International Conference on Computer Vision, 10722-10731 (2019).

45. Rupprecht, C., Huaroc, E., Baust, M. \& Navab, N. Deep active contours. arXiv preprint arXiv:1607.05074 (2016).

46. Ma, D., Liao, Q., Chen, Z., Liao, R. \& Ma, H. Adaptive local-fitting-based active contour model for medical image segmentation. Signal Process. Image Commun. 76, 201-213 (2019). 
47. Fang, J., Liu, H., Zhang, L., Liu, J. \& Liu, H. Fuzzy region-based active contours driven by weighting global and local fitting energy. IEEE Access (2019).

48. Liu, H., Fang, J., Zhang, Z. \& Lin, Y. A novel active contour model guided by global and local signed energy-based pressure force. IEEE Access (2020). 
Figures

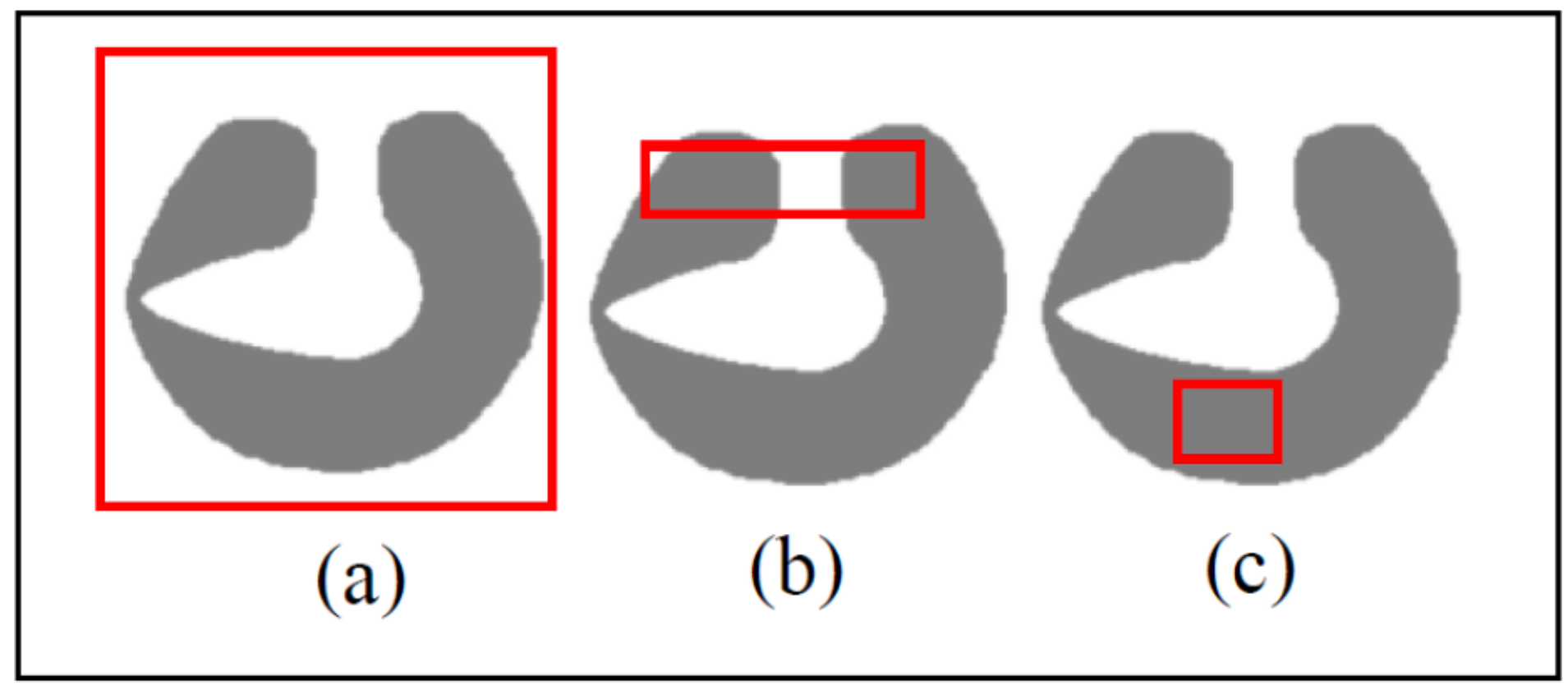

Figure 1

Three examples of initial contours considered outside (a), overlap (b), and inside (c) the image 


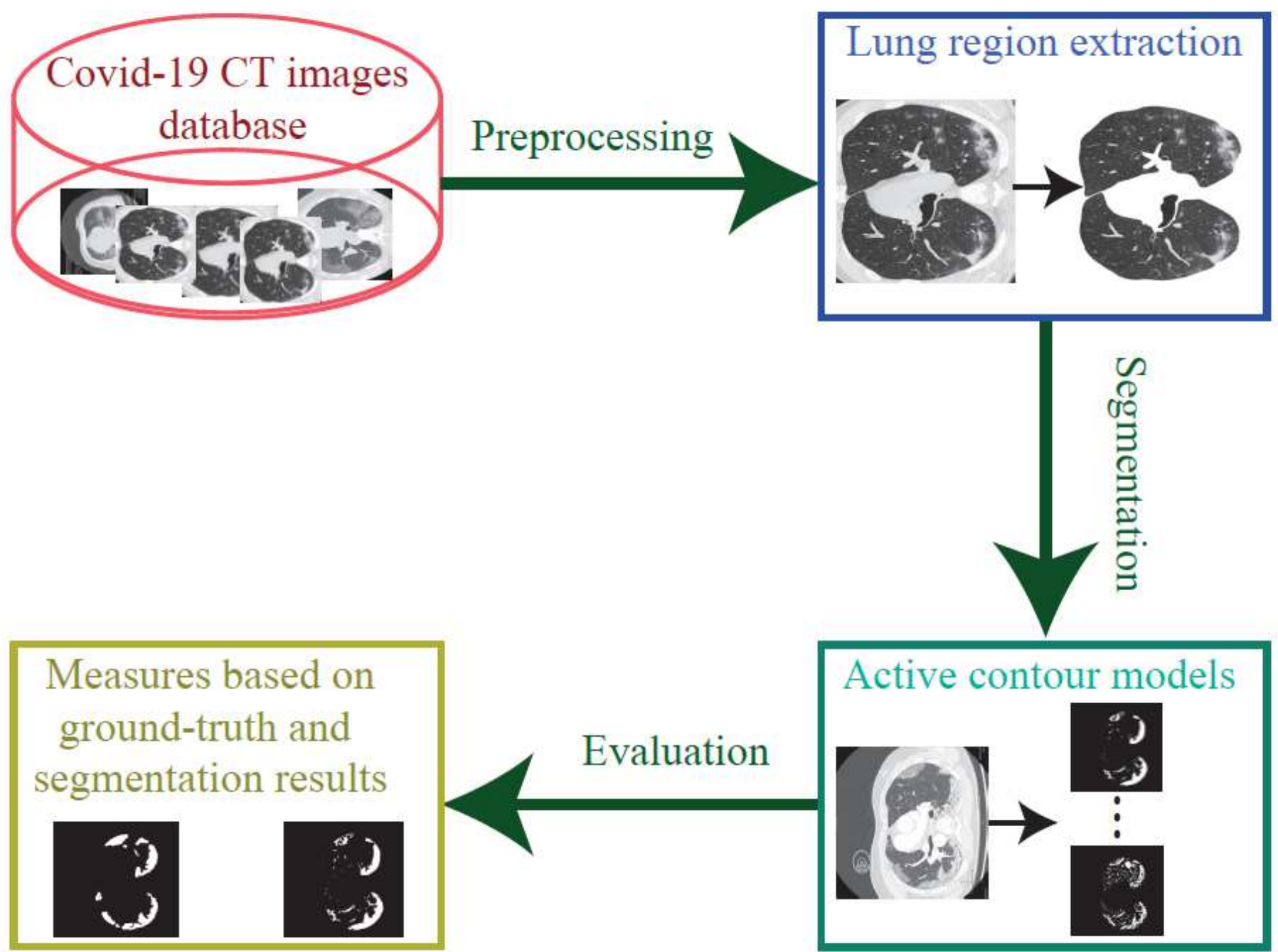

Figure 2

Overview of the proposed steps 


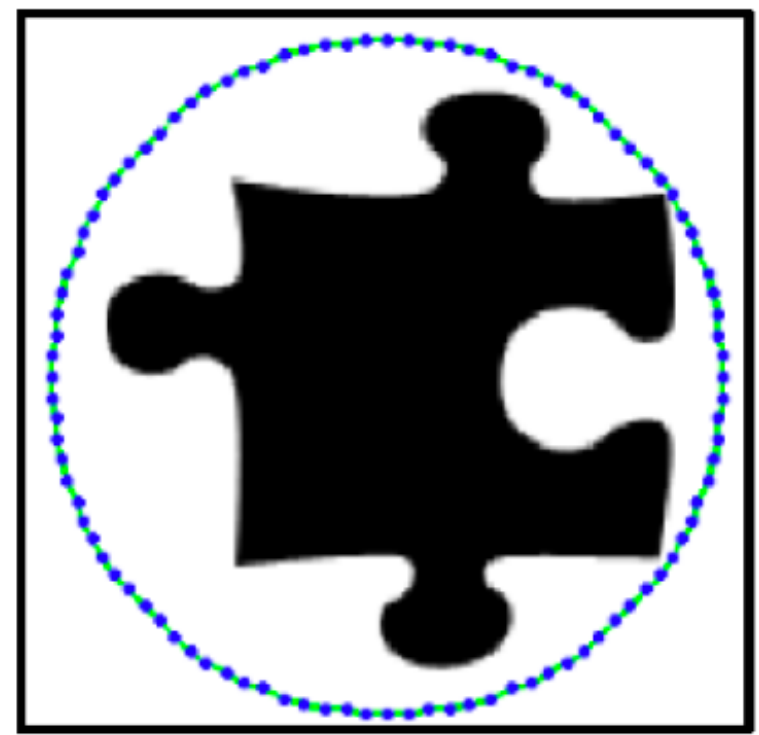

(a)

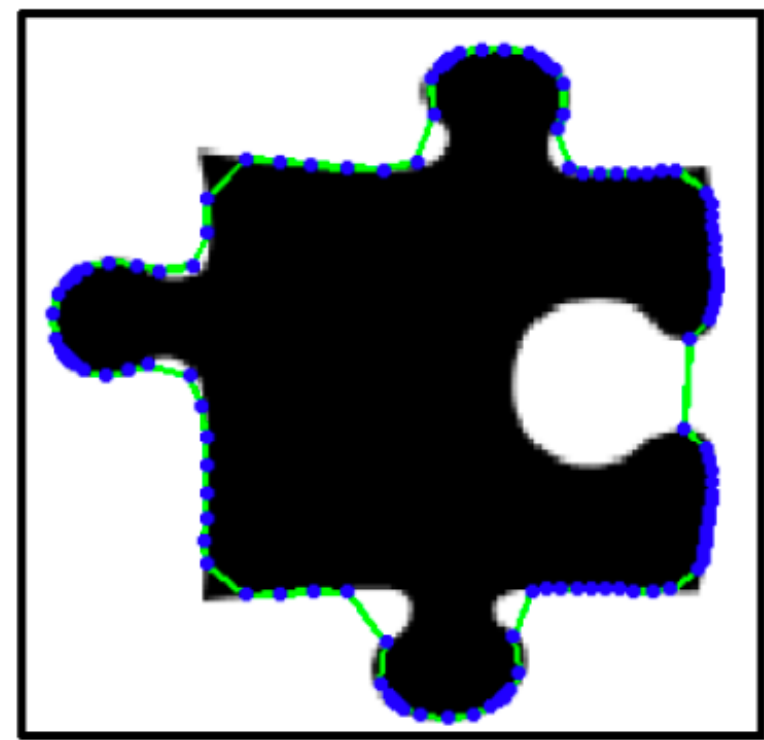

(b)

Figure 3

An example of concave regions; (a) is the initial snake, and (b) is the final state of snake (by Kass) occurred after 2346 iterations

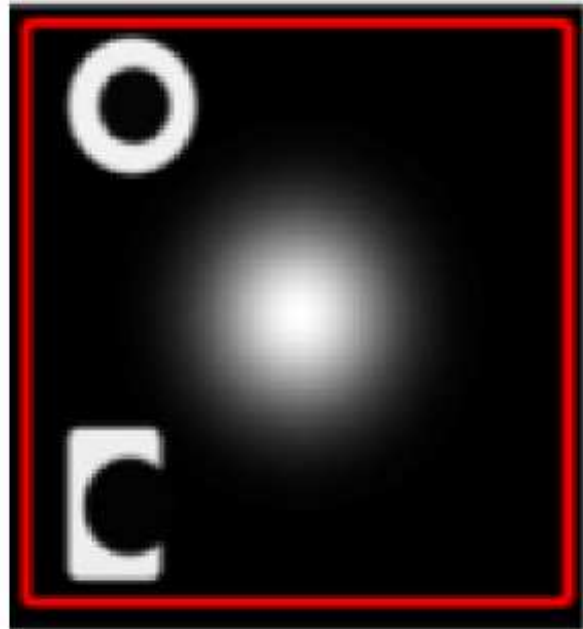

(a)

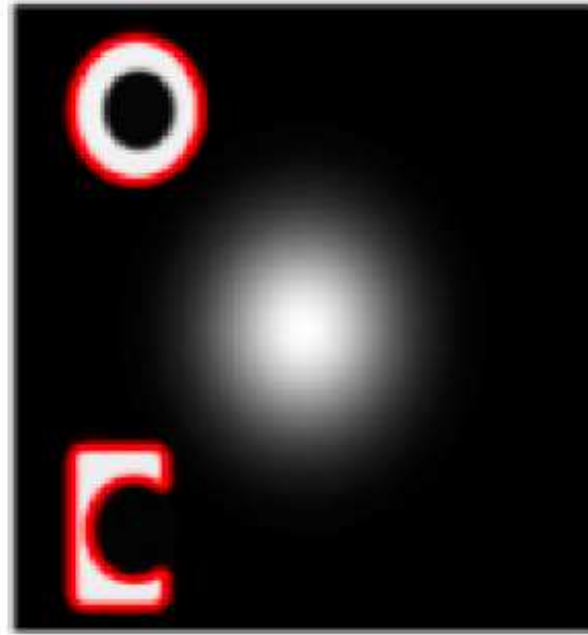

(b)

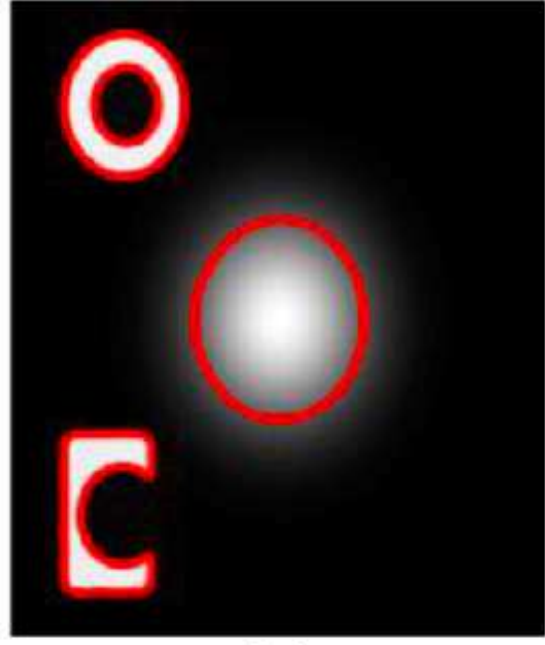

(c)

\section{Figure 4}

Segmentation result on a synthetic image based on GAC model; (a) the initial contour shown in red color, (b) the final segmentation result, and (c) shows all of the boundaries that should be surrounded 

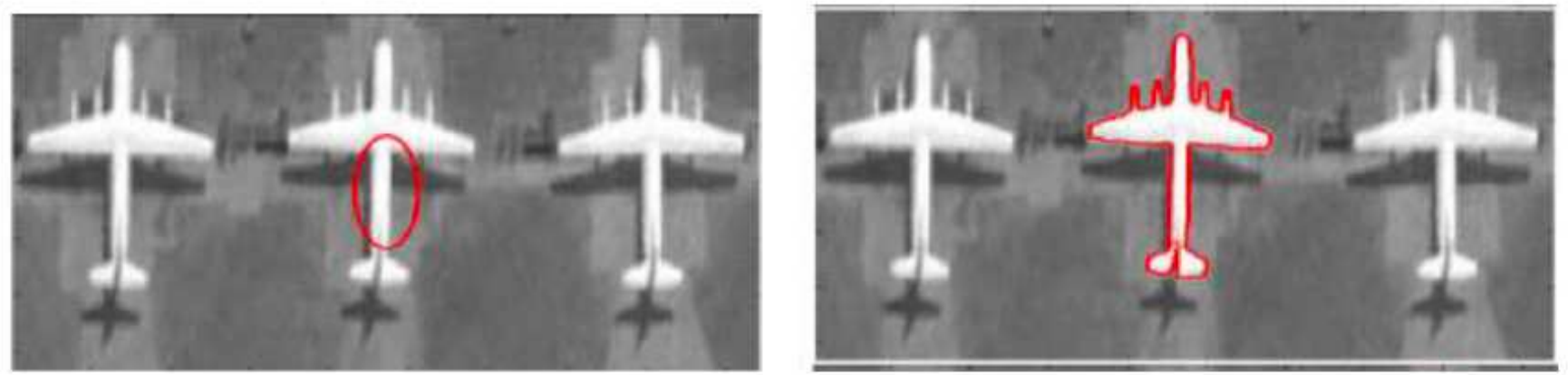

(a)

(b)

Figure 5

An example of global segmentation property of C-V model. (a) shows the initial contour, and (b) shows the segmentation result of $\mathrm{CV}$ method which cannot extract all boundaries in the image 

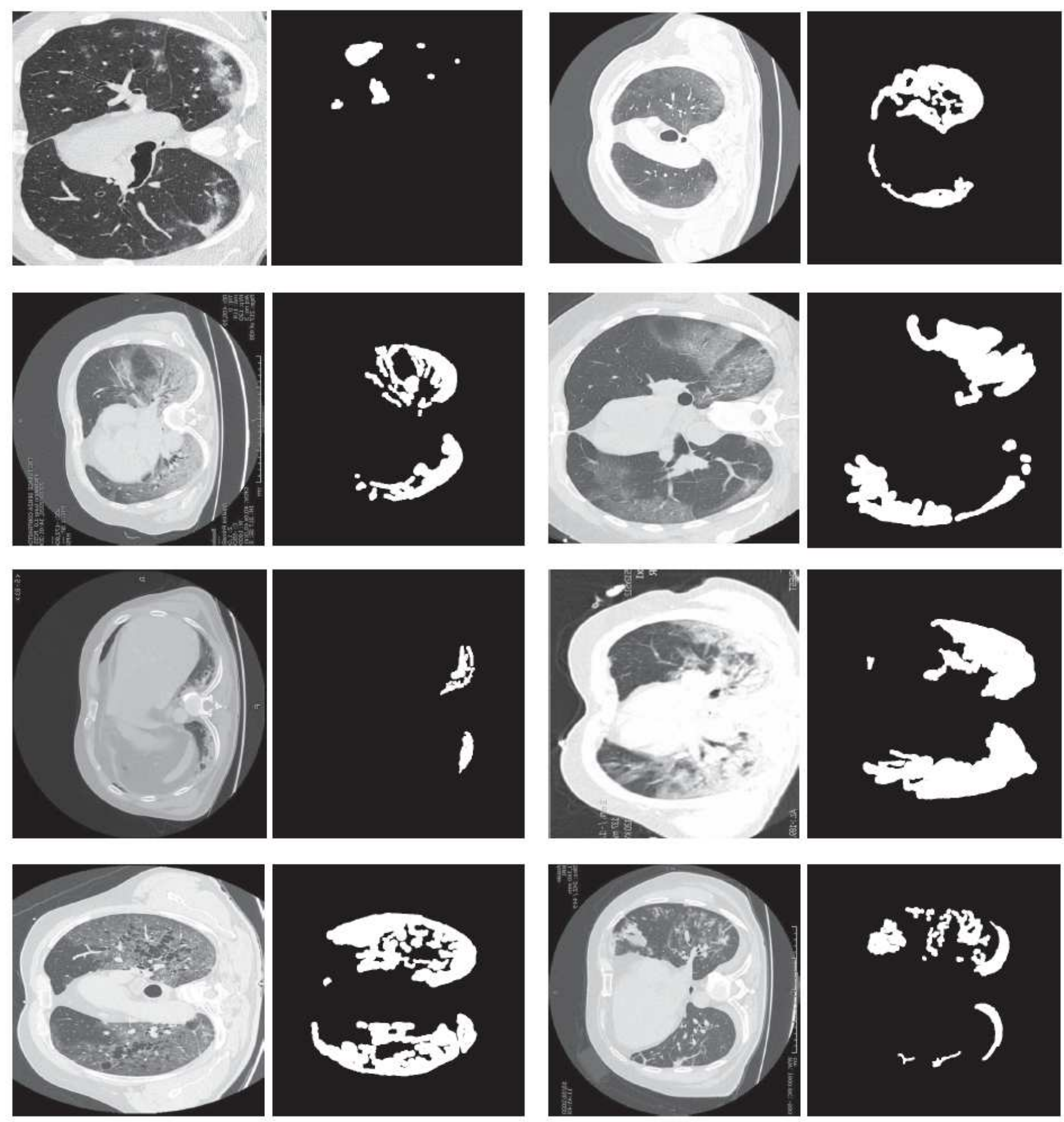

Figure 6

Samples of images of COVID-19 database and their ground truth. 

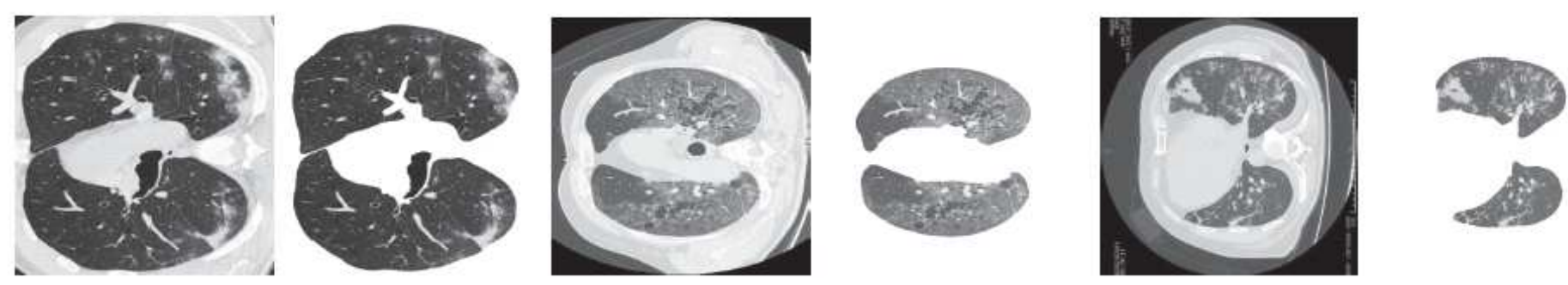

Figure 7

Outcomes of extraction lung region extraction 


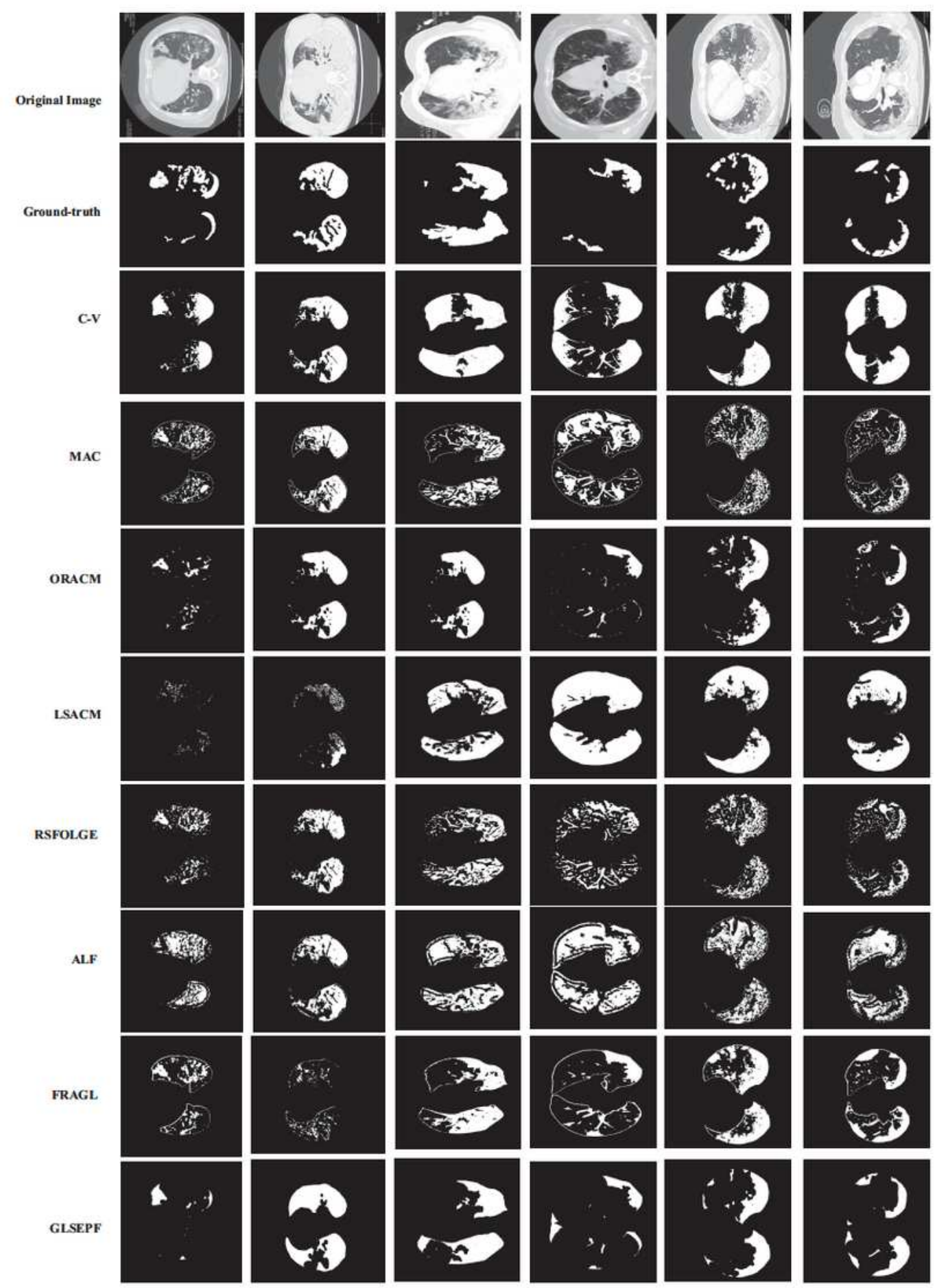

Figure 8. Outcomes of all active contour models in terms of the database.

Figure 8

Outcomes of all active contour models in terms of the database. 